\title{
Permutation Polynomial Interleavers: An Algebraic-Geometric Perspective
}

\author{
Oscar Y. Takeshita \\ Dept. of Electrical and Computer Engineering \\ 2015 Neil Avenue \\ The Ohio State University \\ Columbus, $\mathrm{OH} 43210$ \\ Takeshita.3@osu.edu
}

\author{
Submitted to the IEEE Transactions on Information Theory \\ January 12, 2006
}

\begin{abstract}
An interleaver is a critical component for the channel coding performance of turbo codes. Algebraic constructions are important because they admit analytical designs and simple, practical hardware implementation. The spread factor of an interleaver is a common measure for turbo coding applications. Maximum-spread interleavers are interleavers whose spread factors achieve the upper bound. An infinite sequence of quadratic permutation polynomials over integer rings that generate maximum-spread interleavers is presented. New properties of permutation polynomial interleavers are investigated from an algebraic-geometric perspective resulting in a new non-linearity metric for interleavers. A new interleaver metric that is a function of both the non-linearity metric and the spread factor is proposed. It is numerically demonstrated that the spread factor has a diminishing importance with the block length. A table of good interleavers for a variety of interleaver lengths according to the new metric is listed. Extensive computer simulation results with impressive frame error rates confirm the efficacy of the new metric. Further, when tail-biting constituent codes are used, the resulting turbo codes are quasi-cyclic.
\end{abstract}

\section{Index Terms}

algebraic, geometry, interleaver, permutation polynomial, quadratic, quasi-cyclic, spread, turbo code. 


\section{INTRODUCTION}

Interleavers for turbo codes [1]-[14] have been extensively investigated. However, the design of interleavers for turbo codes is complex enough and we believe there are still several relevant open questions. Recently, Sun and Takeshita [1] suggested the algebraic approach of using permutation polynomial-based interleavers over integer rings. In this paper, we expand the theory in [1] by adding a geometric and group-theoretic perspective. Several new results and important and interesting new questions arise with this framework.

We believe that the approach in [1] has major advantages over any earlier interleaver constructions (either pseudo-random or structured) because it simultaneously provides:

- Excellent error performance with practical code-lengths [15].

- Completely algebraic structure with elegant and relevant properties [15], [16].

- Efficient implementation [17] with high-speed, low-power consumption, and little memory requirements.

\section{A. Interleavers and Permutation Polynomials}

An interleaver is a device that permutes a sequence of $N$ symbols. Let each symbol be indexed by an element in the set $S_{N}=\{0,1, \ldots, N-1\}$. Then the interleaver can be represented by a one-to-one onto (permutation) function $f: S_{N} \rightarrow S_{N}$ given by $f: x \mapsto f(x)$.

Permutation polynomials over $\mathbb{Z}_{N}$ are functions that belong to the ring $\mathcal{R}_{N}=\mathbb{Z}_{N}[x]$, i.e., polynomials of the form $q(x)=\sum_{i=0}^{K} q_{i} x^{i}(\bmod N)$ such that $q_{i} \in \mathbb{Z}_{N}$ and $q: \mathbb{Z}_{N} \rightarrow \mathbb{Z}_{N}$ is a permutation function. In earlier work, we only treated constant-free PPs, i.e., $q_{0}=0$; in this paper, we also consider $q_{0} \neq 0$. The set of permutation functions (not necessarily permutation polynomials) for a given interleaver length $N$ will be denoted by $\mathcal{P}_{N}$. The set $\mathcal{P}_{N}$ is no longer a ring but its elements form a group under function composition.

Permutation polynomials over $\mathbb{Z}_{N}$ naturally generate interleavers where $S_{N}$ gets equipped with a finite ring structure $\mathbb{Z}_{N}$, which is not necessary for the definition of an arbitrary interleaver but allows a useful algebraic treatment of PP interleavers. Replacing $S_{N}$ by $\mathbb{Z}_{N}$ in the case of arbitrary interleavers brings no loss of generality, therefore from here on we will always use $\mathbb{Z}_{N}$. The algebraic structure of PPs over finite integer rings has already been proven very successful in producing turbo codes [15] and low density parity check (LDPC) codes [18] with excellent error performance compared with the best known constructions and similar parameters.

Conditions for the coefficients of a polynomial over $\mathbb{Z}_{N}$ to be a PP for an arbitrary integer $N$ were studied in [1]. However, the conditions are not the simplest for polynomials of degrees larger than two; to the best of our knowledge, simple necessary and sufficient conditions for arbitrary degrees are only known when $N$ is a power of two [19]. If $q(x)$ is of second degree and $N$ is arbitrary then a simple necessary and sufficient condition was proved in [1] and summarized in [15], [16]. Interleavers constructed using second degree or quadratic permutation polynomials will be called QPP interleavers, linear interleavers if the PP is of first degree, and PP interleavers for PPs of arbitrary degrees.

\section{B. Good Interleaver Measures}

Because the number of distinct permutations of length $N$ is $N$ !, it is very important that effective interleaver measures for turbo codes are defined; this considerably reduces the number of interleavers that still need to be filtered by costly analysis and computer simulations for a complex turbo codec system. The main drawback of the theory in [1] was that despite it provided some rules for choosing good permutation polynomials (PP), the procedures were too complex when handling input weights larger than two. In this paper, we propose a new simple but effective measure $\Omega$ for interleavers. Two typical measures for interleavers in turbo coding are the spread factor $D$ [8], [12] and "randomness." In this paper, the notion of "randomness" is replaced by a more principled concept of a degree of non-linearity $\zeta$ of an interleaver. An 
interleaver is represented by what we call an interleaver-code, which is the geometric representation of an interleaver by pairs of coordinates $(x, f(x))$ forming points in $\mathbb{Z}_{N}^{2}$. The degree of non-linearity $\zeta$ measures the number of disjoint orbits (a set of points) of the action of an isometry group of the interleaver-code. The new measure is simply the product of the logarithm of the spread factor by the new non-linearity metric, i.e., $\Omega=\ln (D) \zeta$. The algebraic-geometric nature of PPs allows a very efficient selection of PPs that maximizes the new metric.

DRP interleavers [9] are among the best known interleavers for turbo codes with a combined excellent error rate performance (exceeding that of $S$-random interleavers [12]) and simplicity but are not fully algebraic. The efficacy of the new metric is shown by simulation curves of several turbo codes using PP interleavers with impressive frame error rate performance, similar to the ones with DRP interleavers. However, the selection of good PP interleavers (particularly QPP interleavers) is much simpler with the results presented this paper.

\section{The Relationship between $D$ and $\zeta$}

The non-linearity metric $\zeta$ is shown to be inversely related to the degree of shift-invariance $\epsilon=1 / \zeta$ of an interleaver. For QPP interleavers, the shift-invariance $\epsilon$ is computed in closed form as function of the second degree coefficient, which gives us a complete control of this parameter. When tail-biting codes are used as constituent codes, turbo codes using QPP interleavers become quasi-cyclic; for those codes, it is predicted that the multiplicity of many low-weight codewords is typically multiples of the shift-invariance $\epsilon$. It is also shown that a lower bound on the spread factor $D$ constrained to points within an orbit of an interleaver-code has a closed form for QPP interleavers. An immediate tie between $D$ and $\zeta$ is then established for QPP interleavers where one would need to trade for either larger $D$ or $\zeta$.

This paper is organized as follows. In Section III maximum-spread interleavers are investigated and a list of good permutation polynomials for turbo codes for several lengths that maximizes the spread factor $D$ is found in Table \ A new metric for interleavers is developed in Section 【I, we provide a table (Table III) of good permutation polynomials for turbo codes according to the new metric. Numerical results are shown in Section [V] via the computation of the distance spectra and computer simulations for several codes. Finally, conclusions and possible future directions are discussed in Section $\nabla$.

\section{MAXIMUM-SPREAD INTERLEAVERS}

In this section, the spread factor of an interleaver is revisited. An infinite sequence of QPP interleavers achieving the upper bound on the spread factor is presented. Further, a list of QPP interleavers with optimal spread factors for several interleaver lengths reported in the literature is provided.

\section{A. A Geometric View of Interleavers}

In the algebraic-geometric treatment in this paper, it will be convenient to view an interleaver represented by a permutation function $f(x)$ (not necessarily a permutation polynomial) as an interleaver-code $F$ (not to be confused with a turbo code) under the natural mapping $\Phi: \mathcal{P}_{N} \rightarrow \mathbb{Z}_{N}^{2}$ given by $\Phi: f(x) \mapsto F$, where $F=\left\{(x, f(x)) \mid x \in \mathbb{Z}_{N}\right\}$. We will call a pair $(x, f(x))$ as a point $p_{x}=(x, f(x)) \in F$. Let a linear interleaver be given by the PP $l(x)=31 x(\bmod 512)$; the corresponding interleaver-code $L=\Phi(l)$ is plotted in Fig. 1

The notion of distance or metric between points in an interleaver-code $F$ will be of central role. We will mainly use two different metrics.

1) $L_{1}$ metric: Let us define a distance $\delta$ in $\mathbb{Z}_{N}^{2}$ to form a metric space $\left(\mathbb{Z}_{N}^{2}, \delta\right)$,

$$
\delta\left(p_{x_{1}}, p_{x_{2}}\right)=\left|x_{1}-x_{2}\right|+\left|f\left(x_{1}\right)-f\left(x_{2}\right)\right|,
$$

i.e., the metric is the $L_{1}$ or Manhattan metric. 


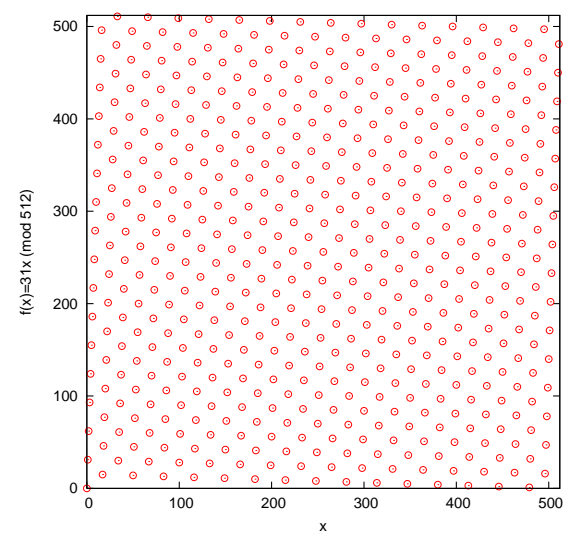

Fig. 1. The interleaver $l(x)=31 x(\bmod 512)$ viewed as an interleaver-code $L=\Phi(l)$ over $\mathbb{Z}_{N}^{2}$.

2) Lee metric: Let us define a distance $\delta_{N}$ in $\mathbb{Z}_{N}^{2}$ to form a metric space $\left(\mathbb{Z}_{N}^{2}, \delta_{N}\right)$,

$$
\delta_{N}\left(p_{x_{1}}, p_{x_{2}}\right)=\left|x_{1}-x_{2}\right|_{N}+\left|f\left(x_{1}\right)-f\left(x_{2}\right)\right|_{N},
$$

where

$$
|i-j|_{N}=\min \{(i-j) \quad(\bmod N),(j-i) \quad(\bmod N)\}
$$

is the Lee distance [20] between $i$ and $j$ modulo $N$. Therefore, $\delta_{N}$ is a two-dimensional Lee metric.

\section{B. The Spread Factor of an Interleaver}

The spread factor [12] of an interleaver is a popular measure of merit in turbo coding applications. The spread factor of an interleaver $f(x)$ over the metric space $\left(\mathbb{Z}_{N}^{2}, \delta\right)$ is defined as

$$
D_{E}(f)=\min _{\substack{i, j \in \mathbb{Z}_{N} \\ i \neq j}}\left\{\delta\left(p_{i}, p_{j}\right) \mid p_{i}, p_{j} \in F\right\} .
$$

The root of this measure is the early recognition in turbo coding that self-terminating information sequences of weight two create low-weight codewords [12]. Divsalar and Dolinar then proposed a construction of linear interleavers achieving spread factors $D_{E}$ equal to or close to $\sqrt{2 N}$. They have concluded that by using linear interleavers, the minimum distance of turbo codes conditioned to weight-two self-terminating information sequences grows roughly as $\sqrt{2 N}$. However, recent results show that the true minimum distance grows asymptotically only at most logarithmically [21] for all interleavers. If we use the metric space $\left(\mathbb{Z}_{N}^{2}, \delta_{N}\right)$ then the following definition of spread factor is also possible [8]:

$$
D(f)=\min _{\substack{i, j \in \mathbb{Z}_{N} \\ i \neq j}}\left\{\delta_{N}\left(p_{i}, p_{j}\right) \mid p_{i}, p_{j} \in F\right\} .
$$

The spread factor $D$ is mathematically more convenient because the metric space $\left(\mathbb{Z}_{N}^{2}, \delta_{N}\right)$ is "isotropic" (in the sense of having no boundaries). It is also more suitable when designing turbo codes with tailbiting convolutional constituent codes [8]. An analytical proof on the upper bound $u b_{D}(N)$ of $D$ was recently shown to be $\sqrt{2 N}$ [22]. Clearly $D \leq D_{E}$ by the definition of $\delta$. We show $u b_{D_{E}}(N)$ is close to $u b_{D}(N)$ by computing a new upper bound $u b_{D_{E}}(N)$. It is a constructive bound for certain values of $N$. See Appendix \for a sketch of a proof.

$$
u b_{D_{E}}(N)= \begin{cases}\frac{2(N-1)}{\sqrt{2 N}-1}, & N=2 p^{2}, \quad p=2,3,4 \ldots \\ \frac{2(N-1)}{\sqrt{2 N-1}-1}, & N=p^{2}+(p-1)^{2}, \quad p=2,3,4, \ldots\end{cases}
$$




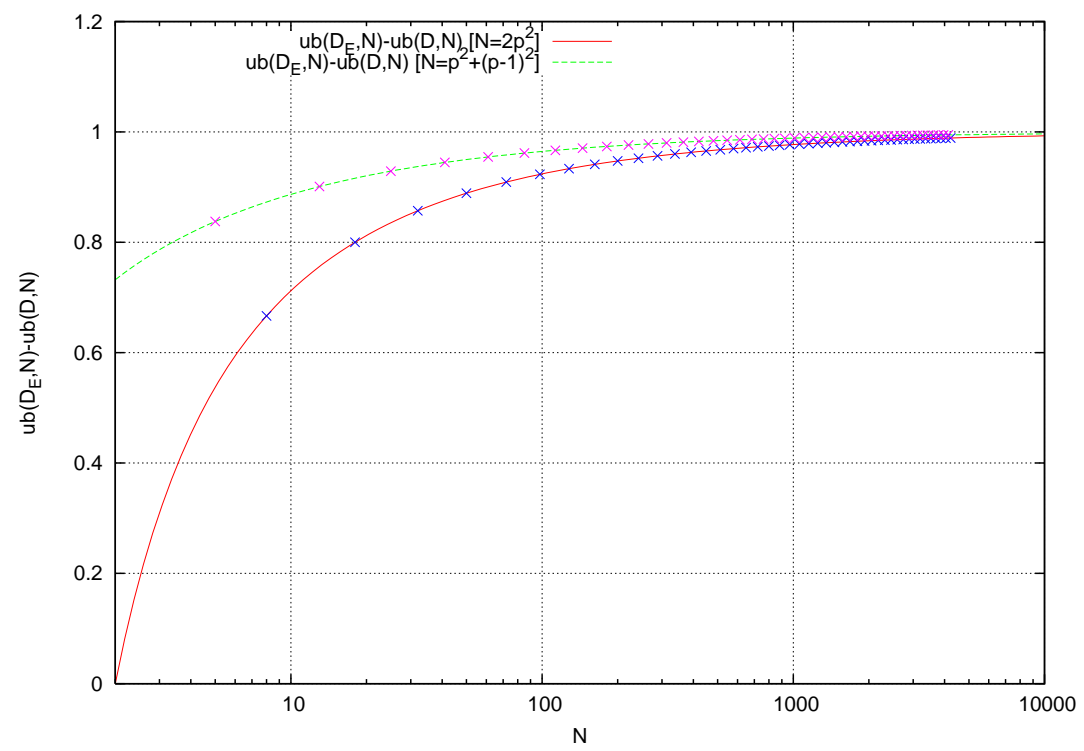

Fig. 2. The difference $u b_{D_{E}}(N)-u b_{D}(N)$.

The difference $u b_{D_{E}}(N)-u b_{D}(N)$ goes to 1 as $N$ grows (See Fig. 2).

Only one example was found (for a limited search) of an interleaver (up to symmetries) that has a spread factor $D_{E}$ exceeding $u b_{D}(4)$. For $N=4$, the interleaver $t: \mathbb{Z}_{4} \rightarrow \mathbb{Z}_{4}$ defined by $t(0)=1, t(1)=3$, $t(2)=0$, and $t(3)=2$ has

$$
u b_{D}(4)=2.8284<D_{E}(t)=3 .
$$

Our new bound (2) is not defined at $N=4$ but for this case clearly an upper bound is $u b_{D_{E}}(4)=3$ by inspection, i.e., an upper bound on $D_{E}$ strictly larger than $u b_{D}(4)$ is achievable. The main message learned is that in practice considering $D$ is good enough. For the remaining of the paper, we will only use $D$ instead of $D_{E}$, unless otherwise noted, because $D$ lets us use the algebra of $\mathbb{Z}_{N}$.

Definition 1: An interleaver of length $N$ is a maximum-spread interleaver if it achieves a spread factor $D$ equal to the upper bound ${ }^{1} \sqrt{2 N}$.

Once more, Dolinar and Divsalar [12] have reported that linear interleavers either achieve or closely approximate a spread factor of $\sqrt{2 N}$ for any $N$. In particular, if $N$ is twice of a perfect square

$$
N=2 n^{2}, \quad n=1,2,3, \ldots,
$$

they have given all maximum-spread linear interleavers of the form $f(x)=f_{1} x\left(\bmod N=2 n^{2}\right)$ by an explicit simple condition for $f_{1}$. However, they pointed out that the resulting interleavers were not good for turbo coding because of their high regularity (see Fig. 1, which corresponds to a plot of a maximum-spread linear interleaver). This fact is also theoretically addressed by the linear interleaver asymptote in [7], which implied the existence of low-weight codewords of input-weight four and a high multiplicity, close to $N$. Therefore they proposed a semi-random interleaver construction algorithm [12], generating the so-called $S$-random interleavers with a parameter $S$, but the algorithm sacrificed the spread factor $D$. The obtained spread factor is typically $D=S+1 \leq \sqrt{N / 2}+1$, i.e., smaller than about $50 \%$ of the upper bound $u b_{D}(N)=\sqrt{2 N}$. An $S$-random interleaver yield turbo codes with very good error rate performances and became the typical benchmark interleaver. One of the main drawbacks of $S$-random interleavers is a costly storage of a sequence of $N$ integers needed to specify the interleaver. Because the construction algorithm

\footnotetext{
${ }^{1}$ Because the spread factor must be an integer, the upper bound is straightforwardly tightened to $\lfloor\sqrt{2 N}\rfloor$. However, this will be of little relevance in this paper and we keep $\sqrt{2 N}$ for simplicity, unless otherwise noted.
} 
relies heavily on a pseudo-random number generator, the sequence has little margin for compression. This characteristic also makes more difficult for an accurate reproduction of the results by others because typically only the parameter $S$ is reported in the literature; however, the repeatability problem is not so critical because in general, for a given parameter $S$, instances of $S$-random interleavers perform similarly for error rates that are not extremely low, which also reflects a good minimum distance of the associated turbo code. Crozier proposed two interleaver constructions [8] that attempt maximization of the spread factor $D$ but avoid or minimize the regularity of linear interleavers: the high-spread construction and the dithered-diagonal construction. The dithered-diagonal interleavers [8] are reported to be maximum-spread for interleaver sizes as in (3) and have large spread factors for others. Dithered-diagonal interleavers require $n=\sqrt{N / 2}$ integer parameters for their definition. An impressive error performance, exceeding the performance of $S$-random interleavers was shown for $N=512$ [8]. This was a great progress in the field of interleaver constructions for turbo codes because it produced a large spread factor $D$ combined with sufficient irregularity to produce an excellent error performance and a much smaller number of integer parameters specifying the interleaver compared with $S$-random interleavers. Subsequently, Crozier and Guinard proposed dithered relatively prime (DRP) interleavers [9]. DRP interleavers also perform very well but require a much smaller number of integer parameters for their specification. Their typical choice for a good performance requires about 10 integers $^{2}$ for their dither parameters $M=R=4$. For an excellent performance, their dither parameters $M=R=8$ require about 18 integers.

\section{The Spread Factor of QPP Interleavers}

Interleavers based on QPP require only 2 integers as parameters with the simplicity of only a simple quadratic congruence as the algorithm to generate the permutation sequence. In fact, QPP interleavers can be implemented in hardware with only additions and comparisons [17]. If we fix $N$, what is the largest spread for a QPP interleaver? An exhaustive search for the largest achievable spread $D^{\max }(N)$ for $2 \leq N \leq 4096$ is shown in Fig. 3. Due to several algebraic and geometric properties of QPP interleavers explained in Section III the exhaustive search is efficiently completed in a very short time on a regular personal computer using Theorem 7 .

A few of the polynomials for some commonly found interleaver lengths in the literature are reported in Table 【 Some of the QPPs in Table \generate very good turbo codes, however, better QPPs that do not simply attempt maximization of the spread factor are provided in Section III-E

Observing once more Fig. 3, at least in the searched range, the fraction of maximum-spread QPP interleavers is very small. The same plot, however, reveals that the majority of QPP interleavers with the largest spreads lie between $u b_{D}(N)=\sqrt{2 N}$ and $\sqrt{N}$ (about $70 \%$ of the upper bound). Recall that $S$-random interleavers typically achieve only $50 \%$ of $u b_{D}(N)$. It is also important that spread factors $D^{\max }(N)$ are shown only when there exist QPP interleavers for a given $N$ in a strict sense, i.e., with irreducible degree, as explained in Section II-E Linear interleavers always exist for any $N$ but this is not true for QPP interleavers [1], [16]. Between $2 \leq N \leq 4096$, there are 1190 values of $N$ that produce a QPP interleaver (roughly 29\%). This raises another question as to what values of $N$ have QPP interleavers in the strict sense.

Theorem 1: Let $N=2^{3} m=8 m$ for some $m \in\{1,2,3, \ldots\}$. Then there exists a quadratic permutation polynomial $f(x)=f_{1} x+f_{2} x^{2}(\bmod N)$ with irreducible degree.

Proof: One simply chooses $f_{2}=2 m$ and $f_{1}=1$, which satisfy the necessary and sufficient conditions for a quadratic permutation polynomial in [1]. The chosen polynomial is not reducible to a linear polynomial because, by Theorem 3 , its degree of non-linearity is $\zeta=2$.

Theorem 1 may be of practical relevance because it guarantees the existence of QPP interleavers for all positive multiples of a typical computer byte size of 8 .

\footnotetext{
${ }^{2}$ The length of the input parameters are more precisely defined in Section IV-B.1
} 


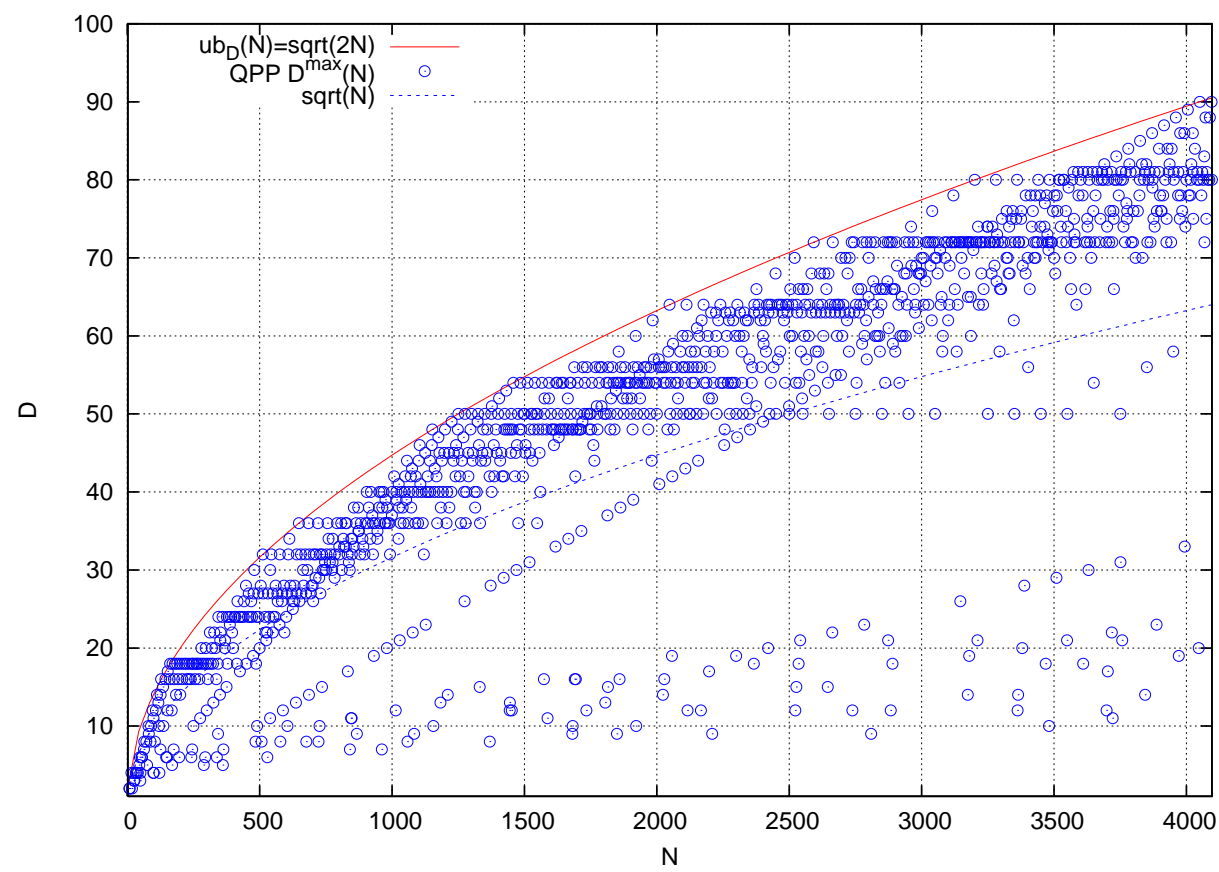

Fig. 3. Maximum achievable spreads $D^{\max }(N)$ with QPP interleavers for a given $N$.

\section{The Maximization of the Spread Factor D}

The maximization of the spread factor $D$ is clearly beneficial in the minimization of the number of lowweight codewords caused by self-terminating weight- 2 input sequences. This concept trivially generalizes to short bursts of self-terminating input sequences, which then include the classical weight- 2 input. Should we then look for interleavers that always maximize the spread factor? To understand the context of this question, we make the following observations:

- Many linear interleavers are maximum-spread but suffer from high-multiplicity low-weight codewords [7].

- At least one dithered diagonal interleaver [8] is maximum-spread and provides an impressive error performance.

- DRP interleavers which maximize error performance are typically not maximum-spread [9].

Although it is not possible to generalize from the single example reported in [8], from observation 2), the best interleavers from an error rate perspective may be the ones that either achieve or closely approximate a maximum-spread interleaver and simultaneously have a large degree of "randomness." Interleavers with some structure such as DRP and our PP interleavers (or low-entropy, as discussed in Section IV-B.11 may need to give up some spread factor in order to convert some of its entropy for "randomness."

A common practice for a more accurate estimation of error performance away from asymptotics is not only to examine the minimum distance of a code but also the distance profile. Similarly, as we better understand PP interleavers, the spread profile may become more important. We define the multiplicities of the spread profile of an interleaver-code $F$ as

$$
A_{D_{i}, p_{x}}=\left|\left\{p_{x_{1}} \mid i=\delta_{N}\left(p_{x}, p_{x_{1}}\right), p_{x} \neq p_{x_{1}}, p_{x}, p_{x_{1}} \in F\right\}\right|,
$$

where $D_{i}, \quad i=1,2, \ldots,\lfloor\sqrt{2 N}\rfloor$, is the $i$-th spread. Good interleaver-codes are in general non-linear (see Section $\amalg$ ), and therefore the spread profile above is a function of each point $p_{x} \in F$. For arbitrary interleavers, computing the spread profile becomes difficult without a brute force search because the interleaver-code induced by $\Phi$ is highly non-linear. However, for PP interleavers, the spread profile can be inspected just for the representatives of the orbits. In fact, what we proposed in [1] can be interpreted as the creation of "spectral nulls" in the spread profile for self-terminating weight- $2 m$ sequences and giving 
TABLE I

QPPS WITH THE LARGEST SPREAD FACTORS $D^{\max }(N)$.

\begin{tabular}{|r|c|c|c|c|c|}
\hline$N$ & $f(x)$ & $f^{-1}(x)$ & $D^{\max }(N)$ & $\zeta$ & $\zeta^{\prime}$ \\
\hline 40 & $x+10 x^{2}$ & $21 x+10 x^{2}$ & 4 & 2 & 2 \\
80 & $9 x+20 x^{2}$ & $49 x+20 x^{2}$ & 10 & 2 & 2 \\
128 & $15 x+32 x^{2}$ & $111 x+32^{2}$ & 16 & 2 & 2 \\
160 & $19 x+40 x^{2}$ & $59 x+40 x^{2}$ & 16 & 2 & 2 \\
256 & $15 x+32 x^{2}$ & $239 x+32 x^{2}$ & 16 & 4 & 3 \\
320 & $19 x+40 x^{2}$ & $59 x+40 x^{2}$ & 20 & 4 & 3 \\
400 & $17 x+100 x^{2}$ & $153 x+100 x^{2}$ & 20 & 2 & 2 \\
408 & $25 x+102 x^{2}$ & $253 x+102 x^{2}$ & 24 & 2 & 2 \\
512 & $31 x+64 x^{2}$ & $479 x+64 x^{2}$ & 32 & 4 & 3 \\
640 & $39 x+80 x^{2}$ & $279 x+80 x^{2}$ & 32 & 4 & 3 \\
752 & $31 x+188 x^{2}$ & $655 x+188 x^{2}$ & 32 & 2 & 2 \\
800 & $17 x+80 x^{2}$ & $753 x+240 x^{2}$ & 32 & 5 & 5 \\
1024 & $123 x+256 x^{2}$ & $691 x+256 x^{2}$ & 34 & 2 & 2 \\
1280 & $39 x+80 x^{2}$ & $279 x+80 x^{2}$ & 40 & 8 & 4 \\
1504 & $183 x+376 x^{2}$ & $263 x+376 x^{2}$ & 46 & 2 & 2 \\
1600 & $49 x+100 x^{2}$ & $849 x+700 x^{2}$ & 50 & 8 & 4 \\
2048 & $63 x+128 x^{2}$ & $1983 x+128 x^{2}$ & 64 & 8 & 4 \\
2560 & $79 x+160 x^{2}$ & $1199 x+160 x^{2}$ & 64 & 8 & 4 \\
3200 & $79 x+800 x^{2}$ & $3119 x+800 x^{2}$ & 80 & 2 & 2 \\
4096 & $173 x+1024 x^{2}$ & $2853 x+1024 x^{2}$ & 80 & 2 & 2 \\
\hline
\end{tabular}

up the maximization of the first spread spectral line $D_{1}$. The shaping of the spread profile may be a promising way for designing and searching for good PPs.

\section{E. An Infinite Sequence of Maximum-Spread QPP Interleavers}

Theorem 2: The following is an infinite sequence of QPPs that generate maximum-spread interleavers.

$$
f(x)=\left(2^{k}-1\right) x+2^{k+1} x^{2} \quad\left(\bmod 2^{2 k-1}\right) \quad k=1,2,3, \ldots
$$

Proof: See Appendix III

Strictly, we have QPP interleavers only when $k>3$. The first observation is that for $k=1$ and $k=2$, the corresponding QPPs $f(x)$ are immediately reduced to first degree polynomials because the second degree coefficient $f_{2} \equiv 0(\bmod N)$. We now show after some preliminaries that for $k=3$, the QPP $f(x)$ is also reducible to a first degree polynomial although $f_{2}=2^{k+1}=16 \not \equiv 0(\bmod N=32)$.

Definition 2: A polynomial $z(x)(\bmod N)$ that evaluates to zero for all $x$, i.e., $z(x) \equiv 0(\bmod N) \quad \forall x$ is called a zero-polynomial.

Proposition 1: Let $N$ be an integer factorable as $N=p q$. The following is a zero-polynomial of degree $p$.

$$
z(x)=m q \prod_{i=0}^{p-1}(x+k+i) \quad(\bmod N) \quad \forall k, m \in \mathbb{Z}_{N}
$$

Proof: Exactly one of the numbers in the sequence $x+k+i, 0 \leq i<p$ is congruent to 0 modulo $p$. Therefore $z(x)$ must evaluate to zero for all $x$ because $m q p \equiv 0(\bmod N)$.

Proposition 2: Let a polynomial $p(x) \bmod N$ and a zero-polynomial $z(x) \bmod N$. Then $s(x) \equiv$ $p(x)+z(x)(\bmod N)$, i.e., $s(x)$ and $p(x)+z(x)$ are equivalent functions modulo $N$.

Proof: This follows directly from the definition of a zero-polynomial.

From Proposition 1] the following is a zero-polynomial of second degree for $N=32$

$$
z(x)=16 x(x+1)=16 x^{2}+16 x \quad(\bmod 32) .
$$


Therefore for $k=3$, by adding $f(x)$ to $z(x)$ we obtain the equivalent first degree polynomial

$$
s(x) \equiv f(x)+z(x)=\left(16 x^{2}+7 x\right)+\left(16 x^{2}+16 x\right) \equiv 23 x \quad(\bmod 32) .
$$

For $k>3$, the polynomials are not reducible to first degree polynomials because they have a degree of non-linearity $\zeta$ larger than 1 as explained in Section 【II (In fact, for $k=1,2,3$ we have $\zeta=1$ ). The first six terms of maximum-spread QPPs that are not reducible to first degree polynomials are shown in Table ㅍ. The last three columns of the table are the degrees of non-linearity $\zeta$, the refined degree of non-linearity $\zeta^{\prime}$ and the degree of shift-invariance $\epsilon$ as explained in Section III.

TABLE II

EXAMPLES OF MAXIMUM-SPREAD $Q P P$ INTERLEAVERS

\begin{tabular}{|c|c|c|c|c|c|c|c|}
\hline$k$ & $N$ & $f(x)$ & $f^{-1}(x)$ & $D=u b_{D}(N)$ & $\zeta$ & $\zeta^{\prime}$ & $\epsilon$ \\
\hline 4 & 128 & $15 x+32 x^{2}$ & $-17 x+32 x^{2}$ & 16 & 2 & 2 & 64 \\
5 & 512 & $31 x+64 x^{2}$ & $-33 x+64 x^{2}$ & 32 & 4 & 3 & 128 \\
6 & 2048 & $63 x+128 x^{2}$ & $-65 x+128 x^{2}$ & 64 & 8 & 4 & 256 \\
7 & 8192 & $127 x+256 x^{2}$ & $-129 x+256 x^{2}$ & 128 & 16 & 7 & 512 \\
8 & 32768 & $255 x+512 x^{2}$ & $-257 x+512 x^{2}$ & 256 & 32 & 12 & 1024 \\
9 & 131072 & $511 x+1024 x^{2}$ & $-513 x+1024 x^{2}$ & 512 & 64 & 23 & 2048 \\
\hline
\end{tabular}

The inverse functions $f^{-1}(x)$ are also provided in Table II The closed form expression for $f^{-1}(x)$ is

$$
f^{-1}(x)=\left(-2^{k}-1\right) x+2^{k+1} x^{2} \quad\left(\bmod 2^{2 k-1}\right) .
$$

One easily verifies that $f\left(f^{-1}(x)\right) \equiv f^{-1}(f(x)) \equiv x(\bmod N)$. For general QPPs, we are not aware of a closed form expression for the inverse functions. Further, not all QPPs have an inverse polynomial that is a QPP. This was first observed in [23]. However, if it exists, it is efficiently computed algebraically using the extended Euclidean algorithm [16]. It is easily verified that the necessary and sufficient condition for the existence of a QPP inverse [16] for the polynomials in Theorem 2 is satisfied.

\section{An Algebraic-Geometric View of InTERLEAVERS}

In this section, PP interleavers are tied to an algebraic-geometric view by examining the isometries of the associated interleaver-codes. A new measure for interleavers arises as a consequence. The measure is easily computed for QPP interleavers using their algebraic-geometric properties. A list of QPP interleavers which maximize the new measure is provided. Finally, some comments on larger degree PPs are made.

\section{A. A New Measure for Interleavers}

The following framework is well known in the context of geometrically uniform codes [24]. A treatment of groups and geometry is found in [25]. A symmetry of a metric space $\mathcal{T}=\left(\mathbb{Z}_{N}^{2}, \delta_{N}\right)$ is a mapping of $\mathcal{T}$ to itself such that the distance between points are preserved. We are only interested in the set of symmetries obtained by translations of the space $\mathbb{Z}_{N}^{2}$ (i.e., circular "slides" in the vertical, horizontal directions and their combinations) because the symmetries obtained by translations are exactly the ones tied to the multiplicity of codewords in a turbo code (other possible, but not allowed, symmetries in this paper are rotations and reflections). The algebraic equivalent of a translation $\mathcal{A}\left(k_{0}, k_{1}\right): \mathbb{Z}_{N}^{2} \rightarrow \mathbb{Z}_{N}^{2}$ that circularly "slides" to the right by $k_{0}$ and upwards by $k_{1}$ is given by

$$
\mathcal{A}\left(k_{0}, k_{1}\right):\left(x_{0}, x_{1}\right) \mapsto\left(x_{0}+k_{0}, x_{1}+k_{1}\right), \quad k_{0}, k_{1} \in \mathbb{Z}_{N}
$$

The set of symmetry functions forms a group $\mathcal{G}$ under function composition. Further, since the only symmetries allowed are translations, $\mathcal{G}$ is clearly a commutative group isomorphic to $C_{N}^{2}$ (the Cartesian product of two cyclic groups of order $N$ ). An isometry of an interleaver-code $F$ is a symmetry $\mathcal{A}$ of $\mathcal{T}$ 
inducing $\mathcal{A}: F \mapsto G$ such that $F=G$. The set of isometries of $F$ form a subgroup $\mathcal{H}$ of $\mathcal{G}$. We say that a point $p_{x_{1}} \in F$ is equivalent to a point $p_{x_{2}} \in F$ when there exists an isometry $\mathcal{A}$ of $F$ that maps $p_{x_{1}}$ to $p_{x_{2}}$.

Definition 3: Let $F$ be an interleaver-code. The orbit of a point $p_{x} \in F$ is the set of points $\mathcal{O}_{p_{x}}$ equivalent under the action of the isometry group $\mathcal{H}$.

Proposition 3: (Theorem 5.1 [25]) There is just one way to express $F$ as the disjoint union of a family of orbits.

Definition 4: The degree of non-linearity $\zeta(F)$ of an interleaver $F$ is the of number of distinct orbits.

Proposition 4: All orbits have the same size.

Proof: This is straightforward from the fact that the only allowed symmetry functions are translations and Proposition 3 .

Definition 5: The degree of shift-invariance $\epsilon(F)$ of an interleaver $F$ is the size of the orbits.

Proposition 5: The degree of non-linearity $\zeta$ and the degree of shift-invariance $\epsilon$ of an interleaver of length $N$ are related by $\zeta=N / \epsilon$.

Proof: This follows directly from their definitions and Proposition 4.

Clearly $1 \leq \zeta, \epsilon \leq N$. Let $l(x)=l_{1} x(\bmod N)$ a linear permutation polynomial inducing an interleavercode $L$ via $\Phi$. A possible symmetry of $L$ is $l(x)=l(x-1)-l_{1}$. Then the degree of non-linearity of $L$ is $\zeta(L)=1$, i.e., the smallest possible. It is reasonable that a randomly chosen permutation function $r(x)$ induces an interleaver-code $R$ via $\Phi: r(x) \mapsto R$ whose degree of non-linearity is likely to be $\zeta(R)=N$, i.e., the largest possible, especially if $N$ is large.

\section{B. The degree of non-linearity of QPP interleavers}

Let us compute the degree of non-linearity of QPP interleavers $q(x)=q_{1} x+q_{2} x^{2}(\bmod N)$ by finding the isometry mappings for the interleaver-code $Q$.

Theorem 3: The degree of non-linearity of a QPP interleaver $Q$ given by $q(x)=q_{1} x+q_{2} x^{2}$ is $\zeta(Q)=$ $N / \operatorname{gcd}\left(2 q_{2}, N\right)$.

Proof: See Appendix IV]

From the proof of the Theorem 3 and because $p_{0}=(0,0) \in Q$ if we assume a constant-free QPP, the set

$$
\mathcal{O}_{p_{0}}=\left\{\left(k_{0}(i), k_{1}(i)\right) \mid i=0,1, \ldots, \operatorname{gcd}\left(2 q_{2}, N\right)-1\right\}
$$

is precisely one of the orbits in $Q$.

Theorem 4: The orbits for the sequence of maximum-spread interleavers in (4) are interpolated by a linear curve.

Proof: This follows from the proof of Theorem 2 where the orbits are exactly intersections between linear curves and the QPP.

Theorem 5: Let $p_{x_{1}}, p_{x_{2}} \in \mathcal{O}_{(0,0)}$ and $p_{x_{1}} \neq p_{x_{2}}$. A lower bound on the distance $\delta_{N}\left(p_{x_{1}}, p_{x_{2}}\right)$ is $2 N \operatorname{gcd}\left(2 q_{2}, N\right)$.

Proof: Half of the distance $2 N \operatorname{gcd}\left(2 q_{2}, N\right)$ is from the minimum distance in set $\left\{k_{o}(i)\right\}$. The other half is from the minimum distance in the set $\left\{k_{1}(i)\right\}$, which turns out to be also equally separated by $\frac{N}{\operatorname{gcd}\left(2 q_{2}, N\right)}$ by the $M C F$ Theorem in [15].

To find the other orbits, we only need one representative from each.

Theorem 6: A complete set of representatives for the distinct orbits of $Q$ is

$$
\left\{(i, f(i)) \mid i=0,1, \ldots, N / \operatorname{gcd}\left(2 q_{2}, N\right)-1\right\} .
$$

Proof: This is so because the orbits are disjoint and to have a point belong to the same orbit, they must be no closer than $N / \operatorname{gcd}\left(2 q_{2}, N\right)$ in either coordinate by Theorem 5] These must cover all representatives because the number of distinct orbits is $\mathrm{N} / \operatorname{gcd}\left(2 q_{2}, N\right)$. 
The decomposition of the interleaver defined by $f(x)=31 x+64 x^{2}(\bmod 512)$ into its four disjoint orbits is shown in Fig. 4
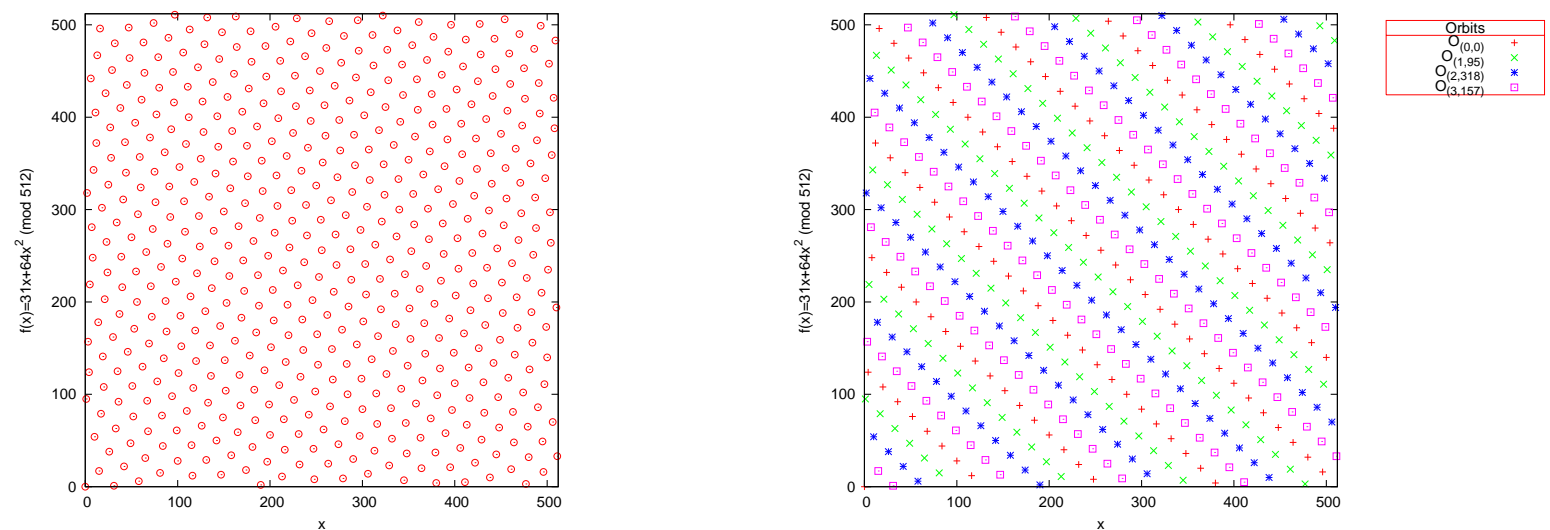

Fig. 4. The interleaver $f(x)=31 x+64 x^{2}(\bmod 512)$ decomposed into its four disjoint orbits.

It is interesting that the placements of the points in both plots in Fig. 4 are exactly the same; however, the regularity (linearity) of the interleaver gets clearly stressed on the right plot.

Definition 6: Let $Q$ be a interleaver-code generated by an arbitrary PP. The local spread of a point $p_{x} \in Q$ is

$$
D_{p_{x}}=\min \left\{\delta_{N}\left(p_{x}, p_{y}\right) \mid \delta_{N}\left(p_{x}, p_{y}\right) \leq \sqrt{2 N}, \sqrt{2 N}\right\} .
$$

We have now the following Theorem on an efficient computation procedure for the spread factor $D$ of PP interleavers.

Theorem 7: Let $Q$ be a interleaver-code generated by an arbitrary PP and let $\left\{p_{x}\right\}$ be a set of representatives for each orbit in $Q$. The spread factor $Q$ can be computed by

$$
D=\min \left\{D_{p_{y}} \mid p_{y} \in\left\{p_{x}\right\}\right\} .
$$

Proof: This follows from the fact that all points in a particular orbit are equivalent under translations and a local spread cannot exceed the upper bound on $D$.

\section{A Refined Non-linearity Metric}

One problem with the non-linearity metric $\zeta$ is that it does not capture the notion of orbits that are disjoint but are "co-linear," i.e., there exists a linear curve interpolating them. We propose another nonlinearity metric $\zeta^{\prime} \leq \zeta$ that fixes part of this problem. A QPP $q(x)=q_{1} x+q_{2} x^{2}(\bmod N)$ can be decomposed in two monomials, one corresponding to the first degree term $q_{1} x(\bmod N)$ and the other to the second degree term $q_{2} x^{2}(\bmod N)$. In most cases, we have $\operatorname{gcd}\left(q_{1}, N\right)=1$ for a valid QPP, which means that $q_{1} x(\bmod N)$ is a linear permutation polynomial (in the case that $\operatorname{gcd}\left(q_{1}, N\right) \neq 1 \mathrm{a}$ generalization is possible). A QPP can therefore be viewed as a linear PP that is "disturbed" ${ }^{3}$ by $q_{2} x^{2}$ $(\bmod N)$ at every position $x$. For example, the point at coordinate $x=0$ gets disturbed by $q_{2} 0^{2}=0$, the point at coordinate $x=1$ gets disturbed by $q_{2} 1^{2}=q_{2}$ and so on. By Theorem 6 , the periodicity of the disturbance is at most $\zeta$. The non-linearity $\zeta^{\prime}$ simply measures how many distinct elements we have in the set $\left\{q_{2} x^{2}(\bmod N) \mid x=0,1, \ldots, \zeta-1\right\}$, which is a very simple measure. The values for $\zeta^{\prime}$ in Table $\square$ reveal that they do not grow as fast as $\zeta$.

\footnotetext{
${ }^{3}$ There is a similarity with DRP interleavers where a linear permutation polynomial is "dithered." However, as argued in Section IV-B.1 DRP and PP interleavers are not the same.
} 


\section{Low-Weight Codewords in Turbo Codes with Uniterleaved Dual Termination}

Parallel concatenated turbo codes using trellis termination such as the one in the 3GPP standard (both trellises are terminated but termination bits in neither constituent codes are interleaved) are known to suffer from low-weight codewords if a weight-one input sequence near the end of one constituent code maps to a near-end position in the other constituent code. This fact is confirmed for 3GPP codes in [26]. Similar effects for larger input weights also happen and will be called in general as edge effects. The termination method will be called uninterleaved dual termination (UDT). To minimize edge effects, the following is desirable to be maximized:

$$
\mathcal{C}=\min _{x \in \mathbb{Z}_{N}} \delta((N-1, N-1),(x, f(x)))
$$

We will call $\mathcal{C}$ the corner merit of an interleaver because geometrically it means avoiding points in the right upper corner of $F$. A lower bound on the corner merit $\mathcal{C}$ is guaranteed for permutation polynomial interleavers with a constant term in the polynomial equal to zero.

Proposition 6: Let the constant coefficient of the permutation polynomial $f(x)$ be zero, then in the interleaver-code $F=\Phi(f),(0,0) \in F$. This implies $\mathcal{C} \geq D-2$.

Proof: This follows directly from the definition of the spread factor $D$ and the placement of one of the points in $F$ at $(0,0)$.

Therefore, a corner merit is automatically guaranteed if the spread factor $D$ is reasonable. The concept is also valid for any interleaver with a spread factor $D$ and one of its points placed at $(0,0)$. For PP interleavers, the corner merit is often improved over the lower bound in Proposition 6 by selecting a proper constant term for the PP, i.e., searching over possible vertical translations of the interleaver-code (it may be further improved by examining all translations of the interleaver-code). It is also possible that optimizing edge effects for input-weight one sequences may degrade edge effects for other low-weight input sequences.

Low-weight codewords induced by UDT must have relatively low multiplicities because they are caused by edge effects. If the interleaver is properly designed, they have little impact at moderate frame error rates. However, if extremely low frame error rates are desired, a removal of edge effects looks necessary. To remove this edge effect, there are two approaches: tail-biting convolutional codes [27] and interleaved dual termination (IDT) [28].

The tail-biting approach implies an additional complexity for encoding and decoding. In [18] a construction for LDPC codes using QPPs was proposed; it is also proved that they are quasi-cyclic. A similar argument that is made in [18] can be made to prove that turbo codes using PPs are quasi-cyclic when tail-biting convolutional codes are used. We also state here without giving details that for tail-biting PP turbo codes, the multiplicities of the low-weight codewords are typically multiples of the degree of shift-invariance $\epsilon$.

A second form of avoiding edge-effects is by using IDT as proposed in [28]. Effectively, what this termination method produces is a sub-code of a tail-biting turbo code by choosing a particular time in the circular code and expurgating codewords that start/end at states other than zero. This costs a reduction in rate, and the re-introduction of edge effects. However, because this is a sub-code of the tail-biting form, the weight spectrum must be better than the "mother" tail-biting code. Further, encoding is also a little more complex than regular turbo encoders but decoding has the same complexity.

\section{E. A Simple Metric for Permutation Polynomial Interleavers}

We introduced in [1] a procedure for the selection of QPPs matched to a choice of a constituent convolutional code that had in mind the elimination of self-terminating input sequences of weight $2 \mathrm{~m}$ for small integer $m$ 's. The procedure is, however, too cumbersome for $m>1$. We propose now a simpler concept that has its objective the maximization of the following metric for an interleaver $f(x)$ :

$$
\Omega(f)=\ln (D(f)) \zeta(f) .
$$


In other words, we want to maximize the product of the logarithm of the spread factor by the degree of non-linearity of the permutation interleaver. The reasoning is that the minimum distance of a turbo code is now known to grow at most logarithmically; therefore the spread factor that controls the effective free distance should be "rewarded" at most logarithmically. The non-linearity $\zeta$ is expected to have a proportional reduction in the multiplicities of low-weight codewords so it is reasonable to leave it as is. The corner merit $\mathcal{C}$ is indirectly considered because $\Omega$ is a factor of $D$ and a lower bound for $\mathcal{C}$ is $D-2$ (See Proposition 6). With this new approach, we step aside from attempting to optimize the interleaver for a particular convolutional code as was done in [1]. This simpler measure becomes more important as we investigate more complex polynomials of larger degrees and larger lengths. However, this measure is still very empirical and further understanding is desirable. As we have defined a refined non-linearity metric $\zeta^{\prime}$, we have a corresponding refined metric

$$
\Omega^{\prime}(f)=\ln (D(f)) \zeta^{\prime}(f) .
$$

Interleavers for turbo codes were originally constructed with random properties with the argument that multiplicities of "bad input weights" are reduced. We believe this is an introduction of steps to remove the notion of a less precise notion of "randomness" in turbo codes to more principled concepts. We generated the new Table 1 of possibly good interleavers using this new metric and the idea of not to being greedy in the maximization of the spread factor (see Section [II-D). The table lists polynomials for which $\Omega^{\prime}$ is maximized and with a spread factor $D$ larger or equal to $\beta u b_{D}(N)$; the associated interleavers will be called $\Omega^{\prime}$ QPP interleavers. The threshold $\beta$ makes sure that the spread factor does not get too small for small block lengths. A reasonable threshold has been determined experimentally. In fact, as the block size increases, $\beta$ is let become smaller; this means a maximization of the spread factor is considered less important for larger block lengths. When multiple polynomials with the same product merit $\Omega^{\prime}$ exist, we list the one with smallest coefficient for $f_{2}$ and then for $f_{1}$. Inverse polynomials that are also QPP are listed when they exist. It is also listed the constant coefficient $f_{0}$ that optimizes the corner merit $\mathcal{C}$ for $g(x)=f(x)+f_{0}$. The inverse polynomial $g^{-1}(x)$ is computed by [16] $g(x)=f^{-1}\left(x-f_{0}\right)$.

TABLE III

QPPS WITH BEST $\Omega^{\prime}$ AND $\beta u b_{D}(N) \leq D$.

\begin{tabular}{|r|r|c|c|r|r|r|c|}
\hline$N$ & $f_{0}$ & $f(x)$ & $f^{-1}(x)$ & $D$ & $\Omega^{\prime}$ & $\zeta^{\prime}$ & $\beta$ \\
\hline 40 & 6 & $x+10 x^{2}$ & $21 x+10 x^{2}$ & 4 & 2.77 & 2 & 0.45 \\
80 & 72 & $9 x+20 x^{2}$ & $49 x+20 x^{2}$ & 10 & 4.61 & 2 & 0.45 \\
128 & 89 & $7 x+16 x^{2}$ & $55 x+16 x^{2}$ & 8 & 6.24 & 3 & 0.45 \\
160 & 115 & $9 x+20 x^{2}$ & $9 x+60 x^{2}$ & 10 & 6.91 & 3 & 0.45 \\
256 & 240 & $15 x+32 x^{2}$ & $239 x+32 x^{2}$ & 16 & 8.32 & 3 & 0.45 \\
320 & 304 & $19 x+40 x^{2}$ & $59 x+40 x^{2}$ & 20 & 8.99 & 3 & 0.45 \\
400 & 375 & $7 x+40 x^{2}$ & $343 x+120 x^{2}$ & 16 & 13.86 & 5 & 0.45 \\
408 & 273 & $25 x+102 x^{2}$ & $253 x+102 x^{2}$ & 24 & 6.36 & 2 & 0.45 \\
512 & 433 & $15 x+32 x^{2}$ & $239 x+32 x^{2}$ & 16 & 11.09 & 4 & 0.45 \\
640 & 549 & $19 x+40 x^{2}$ & $219 x+200 x^{2}$ & 20 & 11.98 & 4 & 0.45 \\
752 & 619 & $23 x+94 x^{2}$ & $327 x+94 x^{2}$ & 26 & 9.77 & 3 & 0.45 \\
800 & 786 & $17 x+80 x^{2}$ & $753 x+240 x^{2}$ & 32 & 17.33 & 5 & 0.45 \\
1024 & 992 & $31 x+64 x^{2}$ & $991 x+64 x^{2}$ & 32 & 13.86 & 4 & 0.45 \\
1280 & 1248 & $39 x+80 x^{2}$ & $279 x+80 x^{2}$ & 40 & 14.76 & 4 & 0.45 \\
1504 & 1463 & $23 x+94 x^{2}$ & - & 26 & 13.03 & 4 & 0.45 \\
1600 & 1169 & $17 x+80 x^{2}$ & $753 x+240 x^{2}$ & 32 & 20.79 & 6 & 0.45 \\
2048 & 1315 & $31 x+64 x^{2}$ & $991 x+64 x^{2}$ & 32 & 24.26 & 7 & 0.30 \\
2560 & 2377 & $39 x+80 x^{2}$ & $2199 x+720 x^{2}$ & 40 & 25.82 & 7 & 0.30 \\
3200 & 2328 & $17 x+80 x^{2}$ & $2353 x+240 x^{2}$ & 32 & 31.19 & 9 & 0.30 \\
4096 & 1332 & $31 x+64 x^{2}$ & $991 x+64 x^{2}$ & 32 & 41.59 & 12 & 0.30 \\
5472 & 3104 & $77 x+114 x^{2}$ & - & 36 & 28.67 & 8 & 0.30 \\
8192 & 1084 & $31 x+64 x^{2}$ & $3039 x+2112 x^{2}$ & 32 & 79.71 & 23 & 0.30 \\
\hline
\end{tabular}




\section{F. Permutation Polynomials of Degrees Larger than Two}

We have mentioned that while there are linear maximum-spread interleavers for every $N=2 n^{2}$, this is not true for quadratic permutation polynomials. For example, if $N=200=2 \times 10^{2}$ there are no maximumspread interleavers of second degree. However, the cubic permutation polynomial $f(x)=59 x+60 x^{2}+20 x^{3}$ (mod 200) generates a maximum-spread interleaver. Further, interleaver lengths not admitting quadratic permutation polynomials at all may have permutation polynomials of larger degrees. For example, if $N=5$, there are no quadratic permutation polynomials but $c(x)=x^{3}(\bmod 5)$ is a cubic permutation polynomial of irreducible degree. This means maybe it is worth investigating polynomials of larger degrees because the complexity of evaluating the polynomials only grows linearly with the degree using Horner's rule [29, page 93]. The cubic polynomial $f(x)=59 x+60 x^{2}+20 x^{3}(\bmod 200)$ can be evaluated at every point $x$ with three multiplications and two additions using $f(x)=(59+(60+20 x) x) x(\bmod 200)$. Further, it would be interesting if an efficient implementation that sequentially generates all evaluations $f(0), f(1), f(2), \ldots$ that requires only additions and comparisons for arbitrary polynomial degrees, generalizing the idea in [17], is possible. To determine the coefficients for a permutation polynomial of an arbitrary degree and an arbitrary $N$, there is an easy sufficient condition by using Theorem 2.3 and Corollary 2.5 in [1].

\section{NUMERICAL RESUlTS}

\section{A. Distance Spectra of some Example Codes}

Algorithms for computing the true distance spectra of turbo codes such as in [30] and [26] are very useful to help analysis. We used the algorithm in [30] to compute the first 20 smallest distances and respective multiplicities for a few turbo codes using interleavers in Tables [V] W We constrained the search for input weights at most 10. The turbo codes are parallel concatenated codes of nominal rate 1/3. We used two types of constituent codes: the 8-state constituent convolutional code with generator matrix $\left[1 \quad\left(1+D+D^{3}\right) /\left(1+D^{2}+D^{3}\right)\right]$, also denoted in octal notation as $(13,15)$ and the 16 -state constituent

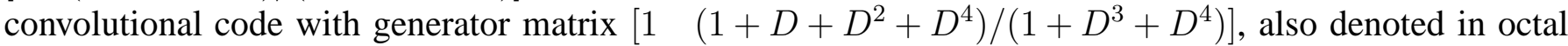
notation as $(23,35)$. The trellis termination method was the same as for the 3rd Generation Partnership Project (3GPP) standard [31], i.e., UDT.

We make the following observations:

- Improving the corner merit $\mathcal{C}$ has mixed effects. It improves the minimum distance (highlighted in the tables) for certain cases but it degrades for others as commented in Section III-D,

- The first spectral line with a high multiplicity has a multiplicity very close to the degree of shift invariance $\epsilon$ as predicted in Section III-D

- The $\Omega^{\prime}$ interleavers appear in general to improve the first spectral line that has a high multiplicity. However, the comparison is not straightforward because the distance spectra is truncated to the first 20 lines.

\section{B. Computer Simulation Results}

The error performances of turbo codes using the maximum-spread QPP interleavers in Table [II well as interleavers in Tables $\square$ and $\amalg$ are examined. The structure of the simulated turbo codes is the same as in the previous section. However, we simulated for both UDT and IDT. The true code-rates for 8-state constituent codes are $N /(3 N+12)$ and $(N-6) / 3 N$ for UDT and IDT, respectively. The true code-rates for 16-state constituent codes are $N /(3 N+16)$ and $(N-8) / 3 N$ for UDT and IDT, respectively.

The true code-rate is used to compute the signal-to-noise ratio $\left(E_{b} / N_{0}\right)$. We used BPSK modulation and assumed an additive white Gaussian noise (AWGN) channel. The decoding was performed with log-MAP decoding and simulated until at least 50 frame errors had been counted, unless otherwise noted. 
TABLE IV

LENGTH $N=128$

\begin{tabular}{|c|c|c|c|c|c|c|c|c|c|c|c|c|c|c|c|c|c|c|c|c|}
\hline \multicolumn{21}{|c|}{8 -state } \\
\hline \multicolumn{21}{|c|}{ MS QPP $f(x)=15 x+32 x^{2}(\bmod N) \epsilon=64$} \\
\hline$i$ & $\mathbf{0}$ & 1 & 2 & 3 & 4 & 5 & 6 & 7 & 8 & 9 & 10 & 11 & 12 & 13 & 14 & 15 & 16 & 17 & 18 & 19 \\
\hline$\overline{\overline{d_{i}}}$ & $\overline{16}$ & $\overline{18}$ & 19 & $\overline{\overline{20}}$ & 21 & 22 & 23 & 24 & 25 & 26 & 27 & 28 & 29 & 30 & 31 & 32 & 33 & 34 & 35 & 36 \\
\hline$N_{i}$ & $\mathbf{1}$ & 1 & 1 & 2 & 55 & 2 & 7 & 8 & 9 & 16 & 27 & 120 & 53 & 359 & 526 & 391 & 775 & 1368 & 1865 & 2542 \\
\hline \multicolumn{21}{|c|}{ 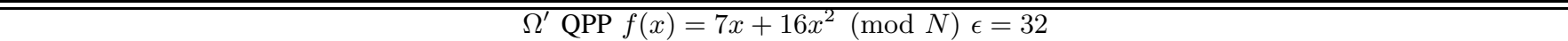 } \\
\hline$i$ & $\mathbf{0}$ & 1 & 2 & 3 & 4 & 5 & 6 & 7 & 8 & 9 & 10 & 11 & 12 & 13 & 14 & 15 & 16 & 17 & 18 & 19 \\
\hline$\overline{d_{i}}$ & $\overline{14}$ & $\overline{15}$ & 16 & $\overline{18}$ & 19 & 20 & 21 & $\overline{22}$ & $\overline{23}$ & 24 & $\overline{25}$ & 26 & 27 & 28 & 29 & 30 & $\overline{31}$ & $\overline{32}$ & $\overline{33}$ & $\overline{34}$ \\
\hline$\overline{N_{i}}$ & 2 & 1 & 1 & 2 & 4 & 3 & 8 & 8 & 33 & 37 & 24 & 123 & 72 & 198 & 111 & 296 & 578 & 731 & 1240 & 1822 \\
\hline \multicolumn{21}{|c|}{$\Omega^{\prime} \mathrm{QPP} f(x)=89+7 x+16 x^{2}(\bmod N) \epsilon=32$} \\
\hline$i$ & $\mathbf{0}$ & 1 & 2 & 3 & 4 & 5 & 6 & 7 & 8 & 9 & 10 & 11 & 12 & 13 & 14 & 15 & 16 & 17 & 18 & 19 \\
\hline$\overline{d_{i}}$ & $\mathbf{1 2}$ & 16 & $\overline{18}$ & 19 & $\overline{20}$ & 21 & 22 & 23 & 24 & 25 & 26 & 27 & 28 & 29 & 30 & 31 & 32 & 33 & 34 & 35 \\
\hline$\overline{N_{i}}$ & $\mathbf{1}$ & 1 & 3 & 2 & 1 & 7 & 8 & 34 & 32 & 15 & 117 & 61 & 184 & 119 & 289 & 532 & 651 & 1166 & 1731 & 3023 \\
\hline \multicolumn{21}{|c|}{ 16-state } \\
\hline \multicolumn{21}{|c|}{ MS QPP $f(x)=15 x+32 x^{2}(\bmod N) \epsilon=64$} \\
\hline$i$ & $\mathbf{0}$ & 1 & 2 & 3 & 4 & 5 & 6 & 7 & 8 & 9 & 10 & 11 & 12 & 13 & $\overline{14}$ & 15 & 16 & 17 & 18 & 19 \\
\hline$\overline{\overline{d_{i}}}$ & $\overline{17}$ & $\overline{18}$ & $\overline{19}$ & $\overline{20}$ & $\overline{21}$ & $\overline{22}$ & $\overline{23}$ & $\overline{24}$ & $\overline{25}$ & $\overline{26}$ & $\overline{27}$ & $\overline{28}$ & $\overline{29}$ & $\overline{\overline{30}}$ & $\overline{\overline{31}}$ & $\overline{32}$ & $\overline{33}$ & $\overline{34}$ & $\overline{\overline{35}}$ & $\overline{36}$ \\
\hline$N_{i}$ & 1 & 3 & 2 & 3 & 1 & 1 & 3 & 1 & 6 & 9 & 14 & 13 & 52 & 28 & 199 & 78 & 79 & 154 & 235 & 422 \\
\hline \multicolumn{21}{|c|}{$\Omega^{\prime}$ QPP $f(x)=7 x+16 x^{2}(\bmod N) \epsilon=32$} \\
\hline$i$ & $\mathbf{0}$ & 1 & 2 & 3 & 4 & 5 & 6 & 7 & 8 & 9 & 10 & 11 & 12 & 13 & 14 & 15 & 16 & 17 & 18 & 19 \\
\hline$\overline{\overline{d_{i}}}$ & $\overline{\mathbf{1 7}}$ & $\overline{21}$ & 23 & $\overline{24}$ & 25 & 26 & $\overline{27}$ & 28 & 29 & 30 & 31 & 32 & 33 & 34 & 35 & 36 & 37 & 38 & 39 & 40 \\
\hline$\overline{N_{i}}$ & $\mathbf{1}$ & 2 & 2 & 2 & 4 & 5 & 31 & 18 & 55 & 23 & 97 & 103 & 133 & 253 & 234 & 596 & $\overline{722}$ & 1261 & 1923 & 3119 \\
\hline \multicolumn{21}{|c|}{$\Omega^{\prime}$ QPP $f(x)=89+7 x+16 x^{2}(\bmod N) \epsilon=32$} \\
\hline$i$ & $\mathbf{0}$ & 1 & 2 & 3 & 4 & 5 & 6 & 7 & 8 & 9 & 10 & 11 & 12 & 13 & 14 & 15 & 16 & 17 & 18 & 19 \\
\hline$\overline{\overline{d_{i}}}$ & 23 & 24 & 25 & 26 & 27 & 28 & 29 & 30 & $\overline{31}$ & 32 & $\overline{\overline{33}}$ & 34 & $\overline{\overline{35}}$ & 36 & 37 & 38 & 39 & 40 & 41 & 42 \\
\hline$\overline{N_{i}}$ & 3 & 1 & 4 & 5 & 31 & 9 & 56 & 16 & 93 & 101 & $\overline{109}$ & $\overline{233}$ & 190 & 577 & 655 & 1099 & 1731 & 2920 & 4407 & 6408 \\
\hline
\end{tabular}

1) Comparison with DRP Interleavers: We compare first length $N=512 \mathrm{PP}$ interleavers under similar conditions with DRP interleavers in [9]. The iterative decoding method used in [9] is simpler than logMAP but worse by about $0.1 \mathrm{~dB}$ at lower SNRs. The DRP curves were obtained directly from [9] but adjusted in SNR to the true code-rate. We used $16 \log$-MAP decoding iterations to match their number of iterations. The simulation curves are compared in Fig. 5

There is a close match in performance with the DRP interleaver with parameter $M=4$ at higher SNRs. The similarity in performance is not quite surprising if we consider that both PP and DRP constructions are based on number congruences. A deeper study of their resemblance is left for a future work, however, we provide a few insights of why QPP interleavers are expected to be close to DRP interleavers with $M=4$ for this interleaver length of $N=512$.

The parameter $M$ in a DRP interleaver is the length of an arbitrary permutation of $M$ letters. Explaining only the essence, a DRP interleaver can be understood as a modification of a linear interleaver by using the permutation of $M$ letters. We show that PP and DRP constructions are in general different, except when $M=1$. When $M=1$, DRP interleavers are what we call linear interleavers, i.e., PPs of first degree because no modification of a linear interleaver is possible if $M=1$. As $M$ grows, DRP interleavers can become arbitrarily "random" in the sense that when $M=N$, arbitrary interleaving functions are possible. The class of PP interleavers cannot generate arbitrary interleaving functions regardless of the degree of the permutation polynomial. This is easily proven by counting arguments but also because all interleavers generated by PPs are maximum contention-free [15] but an arbitrary interleaving function is generally not (maximum) contention-free [2].

Factoring out the construction algorithm, which is similar in complexity and nature, one may characterize PP and DRP interleavers by their input parameters (two vectors of length $M$ for DRP interleavers and the coefficients of the polynomials for PP interleavers). We propose to measure the "randomness" of DRP interleavers by computing the entropy $E$ of the two input permutations of $M$ letters. Considering 
TABLE V

LENGTH $N=512$

\begin{tabular}{|c|c|c|c|c|c|c|c|c|c|c|c|c|c|c|c|c|c|c|c|c|c|}
\hline \multicolumn{22}{|c|}{8 -state } \\
\hline \multicolumn{22}{|c|}{ MS QPP $f(x)=31 x+64 x^{2}(\bmod N) \epsilon=128$} \\
\hline$i$ & $\mathbf{0}$ & 1 & 2 & 3 & 4 & 5 & 6 & 7 & 8 & & 9 & 10 & 11 & 12 & 13 & 14 & 15 & 16 & 17 & 18 & 19 \\
\hline$d_{i}$ & 27 & 28 & 29 & 30 & 31 & 32 & 32 & 34 & 3 & & 6 & 37 & 38 & 39 & 40 & 41 & 42 & 43 & 44 & 45 & 46 \\
\hline$\overline{N_{i}}$ & $\mathbf{1}$ & 2 & 5 & 1 & 8 & 3 & 12 & 4 & 14 & & 4 & 17 & 960 & 1923 & 41 & 304 & 99 & 730 & 1113 & 539 & 406 \\
\hline \multicolumn{22}{|c|}{$\Omega^{\prime}$ QPP $f(x)=15 x+32 x^{2}(\bmod N) \epsilon=64$} \\
\hline$i$ & $\overline{0}$ & 1 & 2 & 3 & 4 & 5 & 6 & 7 & 8 & & 9 & 10 & 11 & 12 & 13 & 14 & 15 & 16 & 17 & 18 & 19 \\
\hline$\overline{\overline{d_{i}}}$ & $\overline{16}$ & $\overline{21}$ & $\overline{22}$ & 24 & $\overline{25}$ & 26 & 27 & 28 & 2 & & 0 & 31 & 32 & 33 & 34 & 35 & 36 & 37 & 38 & 39 & 40 \\
\hline$\overline{N_{i}}$ & $\mathbf{1}$ & 1 & 1 & 1 & 4 & 2 & 2 & 3 & 5 & & 7 & 11 & 13 & 18 & 16 & 36 & 30 & 114 & 200 & 665 & 543 \\
\hline \multicolumn{22}{|c|}{$\Omega^{\prime}$ QPP $f(x)=433+15 x+32 x^{2}(\bmod N) \epsilon=64$} \\
\hline$i$ & $\mathbf{0}$ & 1 & 2 & 3 & 4 & 5 & 6 & 7 & 8 & & 9 & 10 & 11 & 12 & 13 & 14 & 15 & 16 & 17 & 18 & 19 \\
\hline$d_{i}$ & 20 & 21 & 24 & 25 & 26 & 27 & 28 & 29 & 30 & & 11 & 32 & 33 & 34 & 35 & 36 & 37 & 38 & 39 & 40 & 41 \\
\hline$N_{i}$ & 1 & 1 & 1 & 5 & 2 & 1 & 2 & 5 & 9 & & 5 & 9 & 13 & 22 & 27 & 34 & 114 & 196 & 669 & 530 & 430 \\
\hline \multicolumn{22}{|c|}{ Degree-6 PP 512} \\
\hline$i$ & $\overline{\mathbf{0}}$ & 1 & 2 & 3 & 4 & 5 & 6 & 7 & 8 & & 9 & 10 & 11 & 12 & 13 & 14 & 15 & 16 & 17 & 18 & 19 \\
\hline$\overline{\overline{d_{i}}}$ & 27 & 28 & 29 & 30 & 31 & 32 & 3. & 34 & 3 & & 36 & 37 & 38 & 39 & 40 & 41 & 42 & 43 & 44 & 45 & 46 \\
\hline$\overline{N_{i}}$ & 2 & 2 & 5 & 1 & 5 & 2 & 9 & 3 & 12 & & 46 & 79 & 505 & 563 & 58 & 76 & 371 & 617 & 432 & 591 & 1324 \\
\hline \multicolumn{22}{|c|}{16 -state } \\
\hline \multicolumn{22}{|c|}{ MS QPP $f(x)=31 x+64 x^{2}(\bmod N) \epsilon=128$} \\
\hline$i$ & $\mathbf{0}$ & 1 & 2 & 3 & 4 & 5 & 6 & 7 & 8 & & 9 & 10 & 11 & 12 & 13 & 14 & 15 & 16 & 17 & 18 & 19 \\
\hline$d_{i}$ & 26 & 27 & 29 & 31 & 33 & $\overline{34}$ & 3 & 36 & 3 & & 38 & 39 & 40 & 41 & 42 & 43 & 44 & 45 & 46 & 47 & 48 \\
\hline$N_{i}$ & 2 & 1 & 1 & 1 & 3 & 1 & 4 & 3 & 3 & & 4 & 2 & 7 & 14 & 9 & 18 & 25 & 28 & 41 & 151 & 184 \\
\hline \multicolumn{22}{|c|}{$\Omega^{\prime}$ QPP $f(x)=15 x+32 x^{2}(\bmod N) \epsilon=64$} \\
\hline$i$ & $\mathbf{0}$ & 1 & 2 & 3 & 4 & 5 & $\overline{6}$ & 7 & 8 & & 9 & 10 & 11 & 12 & 13 & 14 & 15 & 16 & 17 & 18 & 19 \\
\hline$\overline{d_{i}}$ & $\overline{17}$ & 19 & 23 & 25 & 26 & 27 & 28 & 29 & 30 & & 31 & 32 & 33 & 34 & 35 & 36 & 37 & 38 & 39 & 40 & 41 \\
\hline$N_{i}$ & 1 & 1 & 1 & 1 & 1 & 1 & 1 & 1 & 1 & & 4 & 1 & 3 & 3 & 2 & 12 & 5 & 14 & 16 & 13 & 17 \\
\hline \multicolumn{22}{|c|}{$\Omega^{\prime}$ QPP $f(x)=433+15 x+32 x^{2}(\bmod N) \epsilon=64$} \\
\hline$i$ & $\mathbf{0}$ & 1 & 2 & 3 & 4 & 5 & 6 & 7 & 8 & & 9 & 10 & 11 & 12 & 13 & 14 & 15 & 16 & 17 & 18 & 19 \\
\hline$d_{i}$ & $\overline{18}$ & 19 & $\overline{23}$ & 25 & 26 & 27 & $2 \xi$ & 29 & 3 & & 32 & 33 & 34 & 35 & 36 & 37 & 38 & 39 & 40 & 41 & 42 \\
\hline$N_{i}$ & $\mathbf{1}$ & 1 & 1 & 1 & 1 & 2 & 1 & 1 & 4 & & 1 & 6 & 2 & 3 & 7 & 6 & 14 & 19 & 11 & 16 & 16 \\
\hline \multicolumn{22}{|c|}{ Degree-6 PP 512} \\
\hline$i$ & $\mathbf{0}$ & 1 & 2 & 3 & 4 & 5 & 6 & 7 & 8 & & 9 & 10 & 11 & 12 & 13 & 14 & 15 & 16 & 17 & 18 & 19 \\
\hline$\overline{d_{i}}$ & 27 & 28 & 29 & 30 & 31 & 33 & 35 & 36 & 3 & & 38 & 39 & 40 & 41 & 42 & 43 & 44 & 45 & 46 & 47 & 48 \\
\hline$N_{i}$ & 2 & 1 & 2 & 1 & 1 & 3 & 6. & 1 & 3 & & 6 & 3 & 1 & 6 & 12 & 10 & 19 & 21 & 33 & 45 & 48 \\
\hline
\end{tabular}

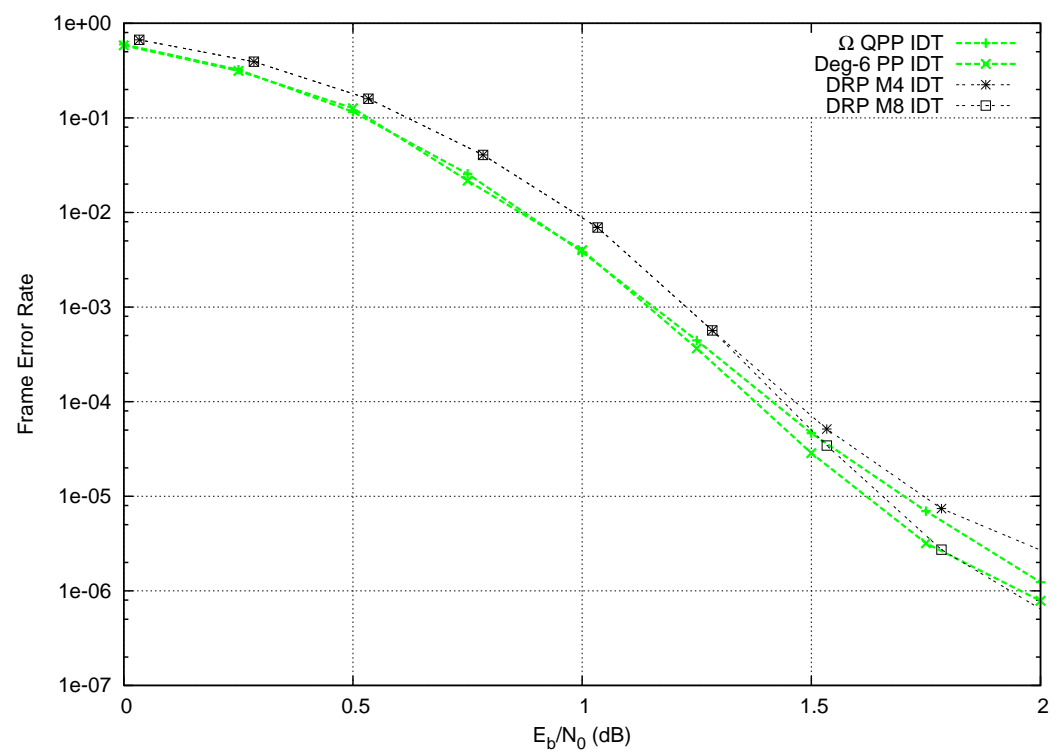

Fig. 5. Length $N=512$ PP interleavers compared with DRP interleavers in turbo codes with 8-state constituent convolutional codes and 16 decoding iterations. 
that an arbitrary sequence of $M$ letters can be chosen, then the entropy is $2 M \log _{2}(M)$ bits, which is independent of the interleaver length. For an interleaver length of $N$, the entropy of the coefficients in $q(x)=q_{1} x+q_{2} x^{2}(\bmod N)$ is $\operatorname{deg}(q(x)) \log _{2}(N / 2)$ bits assuming that $N$ is a power of 2 and $q_{i}$ 's are either even or odd [1]. Now observing the previous matching in Fig. 5] for DRP $(M=4)$ and QPP, we have $E(D R P)=8 \log _{2}(4)=16$ bits and $E(Q P P)=2 \log _{2}(256)=16$ bits with a perfect match. ${ }^{4}$ Would this be a coincidence? The DRP interleaver with $M=8$ has an entropy of $16 \log _{2}(8)=48$ bits, which would correspond to a degree-6 PP. We obtained a very close error performance with a degree six permutation polynomial.

$$
f(x)=15 x+16 x^{2}+128 x^{3}+32 x^{4}+32 x^{5}+64 x^{6} \quad(\bmod 512)
$$

The polynomial in (7) has a degree of non-linearity $\zeta=8$, a refined degree of non-linearity $\zeta^{\prime}=6$ and the orbit size is $\epsilon=64$. Its spread factor is $D=26$ and has a refined product merit of $\Omega^{\prime}=19.55$. The corresponding performance curve is shown in Fig. 5. We used interleaved dual termination (IDT) [28] for this code, which gave a small advantage over UDT at high SNRs (it is not shown in the plot but about a factor of 2 at $2.0 \mathrm{~dB}$ ).

In summary:

- The maximum-spread interleaver $\left(f(x)=31 x+64 x^{2}\right)$ is mostly indifferent to the termination methods and very similar in performance to the DRP $M=4$ and IDT.

- The $\Omega^{\prime}$ interleaver $\left(f(x)=15 x+32 x^{2}\right)$ has a significant improvement with IDT over UDT and is a little better than the maximum-spread code.

- The degree-6 interleaver with IDT and DRP $M=8$ interleaver have mostly the same performance.

2) Performance Curves for 8-State Constituent Codes: We plot next the FER curves for the first four maximum-spread interleavers in Table 1 using UDT and the corresponding constant-free codes in Table III using IDT for turbo codes using the 8-state $(13,15)$ convolutional codes and eight decoding iterations. Similarly as in [15], excellent error performance is obtained down to at least an FER of $10^{-4}$. There is noticeable improvement in FER for codes using $\Omega^{\prime}$ interleavers as the block length increases. Also, a truncated union bound is plotted using the 20 first spectral lines for two of the codes using maximumspread interleavers (MS QPP $N=128$ and MS QPP $N=512$ ).

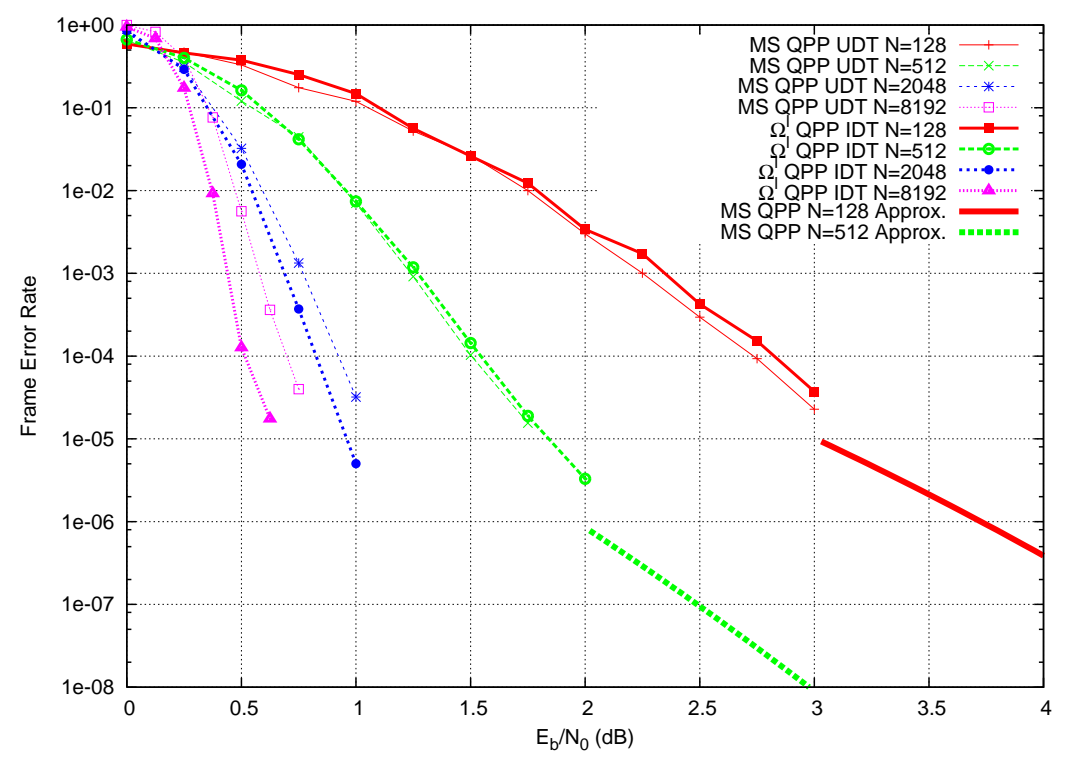

Fig. 6. FER curves for turbo codes with maximum-spread QPP interleavers, 8-state convolutional codes, and eight decoding iterations.

\footnotetext{
${ }^{4}$ It may be argued that the input complexity of DRP interleavers is $\log _{2}(N)$ higher because it requires the specification of a base linear interleaver that includes a constant coefficient.
} 
The refined product merit effectively avoids the selection of bad interleavers. For example, the permutation polynomial

$$
31 x+64 x^{2}+64 x^{3}+32 x^{4}+64 x^{5}+32 x^{6} \quad(\bmod 512)
$$

has a spread factor $D=32$ (a maximum-spread interleaver), a degree of non-linearity $\zeta=8$, a simple product measure of $\Omega=27.73$ but a refined degree of non-linearity $\zeta^{\prime}=2$, and a refined product measure of $\Omega^{\prime}=6.93$. The simulation performance at $1.75 \mathrm{~dB}$ (not shown in plots) gives an FER of $6 \times 10^{-5}$, clearly inferior to the maximum-spread interleaver of second degree that has a larger $\zeta^{\prime}=3$.

3) Performance Curves for 16-State Constituent Codes: An impressive error performance [32] with FERs around $3 \times 10^{-8}$ at an SNR of $1.25 \mathrm{~dB}$ was demonstrated for a DRP interleaver with $M=8$ in a turbo code using the $(23,35) 16$-state constituent code, IDT, and 16 decoding iterations. We show that a similar result is obtained with the QPP interleaver $f(x)=1463+23 x+94 x^{2}(\bmod 1504)$ in Table IIII with the same constituent code, UDT, and 16 log-MAP decoding iterations. We have not simulated the constant-free case with IDT but it should provide an even better error performance. Other codes with interleavers in Tables \(MS) and Tables $\amalg\left(\Omega^{\prime}\right)$ were also simulated.

A genie stopper for the decoding iterations (the iterations are stopped when the decoded information sequence completely agrees with the transmitted one) was used when simulating points at very low FERs. For the length $N=8192 \Omega^{\prime}$ QPP code, at $0.5 \mathrm{~dB}$, close to 6 million frames were simulated with no frame errors with a regular turbo decoder; additionally, almost 40 million frames were simulated with a genie stopper for the iterations, resulting in only three frame errors. Similarly, other points at very low FERs were simulated with the decoding parameters in Table VI]

TABLE VI

Simulation PARAMETERS FOR LOW FERS IN FIG.7

\begin{tabular}{|c|c|c|}
\hline \multicolumn{3}{|c|}{$N=512$} \\
\hline & Frames & Frame Errors \\
\hline Regular & $74,151,303$ & 1 \\
\hline Genie & $657,736,347$ & 7 \\
\hline Total & $731,887,650$ & 8 \\
\hline \multicolumn{3}{|c|}{$N=512 \quad$ MS QPP } \\
\hline & Frames & Frame Errors \\
\hline Regular & $56,273,401$ & 10 \\
\hline Genie & 0 & 0 \\
\hline Total & $56,273,401$ & 10 \\
\hline \multicolumn{3}{|c|}{$N=512 \quad \Omega^{\prime}$ QPP IDT 2.00dB } \\
\hline & Frames & Frame Errors \\
\hline Regular & $195,667,074$ & 11 \\
\hline Genie & & 0 \\
\hline Total & $195,667,074$ & 11 \\
\hline \multicolumn{3}{|c|}{$N=1504 \quad \Omega^{\prime}$ QPP UDT $1.25 \mathrm{~dB}$} \\
\hline & Frames & Frame Errors \\
\hline Regular & $25,603,477$ & 1 \\
\hline Genie & $180,873,414$ & 3 \\
\hline Total & $206,476,891$ & 4 \\
\hline \multicolumn{3}{|c|}{$N=8192 \quad \Omega^{\prime}$ QPP IDT 0.50dB } \\
\hline & Frames & Frame Errors \\
\hline Regular & $5,887,701$ & 0 \\
\hline Genie & $39,323,725$ & 3 \\
\hline Total & $45,211,426$ & 3 \\
\hline \multicolumn{3}{|c|}{$N=8192 \quad$ MS QPP IDT 0.50dB } \\
\hline & Frames & Frame Errors \\
\hline Regular & $4,299,436$ & 0 \\
\hline Genie & $29,305,054$ & 3 \\
\hline Total & $33,604,490$ & 3 \\
\hline
\end{tabular}




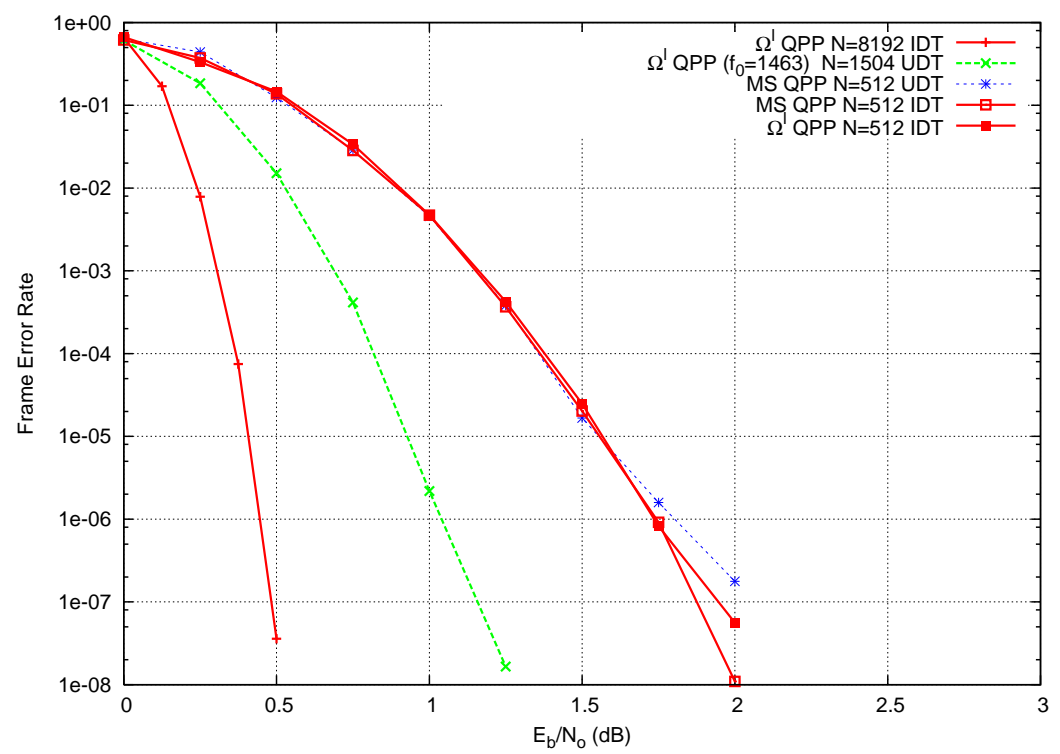

Fig. 7. FER curves for turbo codes with QPP interleavers, 16-state constituent convolutional codes, and 16 decoding iterations.

Although there was an evident improvent by using $\Omega^{\prime}$ polynomials for larger block lengths using 8-state constituent codes, there is not a clear difference for the length $N=8192$ using 16-state constituent codes within the reach of simulations.

In addition, we observe the following for length $N=512$ :

- Codes using both the maximum-spread interleaver $\left(f(x)=31 x+64 x^{2}\right)$ and the $\Omega^{\prime}(f(x)=15 x+$ $32 x^{2}$ ) interleaver benefit significantly with IDT.

- The code with the maximum-spread interleaver and IDT is better than the code with $\Omega^{\prime}(f(x)=$ $\left.15 x+32 x^{2}\right)$.

- The code with the maximum-spread interleaver and IDT has a performance very close to the best interleaver in [8], a dithered diagonal interleaver with identical spread factor of $D=32$.

In summary, for resource-constrained applications, the combination of 8-state constituent codes, a maximum of 8 decoding iterations and QPP interleavers give excellent performance down to FERs around $10^{-4}$ or smaller from short to medium information block sizes of up to 8192 bits. If 16-state constituent codes and a maximum of 16 decoding iterations are acceptable then QPP interleavers give impressive performance down to FERs close to $10^{-8}$ with little or no signs of error-floors.

\section{CONCLUSIONS}

The recently proposed construction of PP interleavers [1] and the subclass of QPP interleavers yield very good error rate performance for turbo codes in a number of practical examples [15], [17]. Interesting properties of QPP interleavers have been studied earlier [1], [15], [16]; however, their characterization is still in its infancy with many open questions. In this paper, the spread factor of QPP interleavers was studied. An infinite sequence of QPP interleavers achieving the maximum-spread was given. Moreover, several properties of PPs and effective measures that are relevant for turbo coding were investigated. We proposed a new refined product measure $\Omega^{\prime}$ for PP interleavers that is easily and quickly computed; a table of good interleavers according to the new measure is provided for several of the interleaver lengths reported in the literature. QPPs are convenient because of their small footprint; however, we provided some evidence that PPs with larger degrees than two may be necessary for very low FERs. Although, turbo codes are limited by a logarithmic growth on the minimum distance [21], that result is asymptotic: good codes at practical lengths achieving FERs close to $10^{-8}$ without signs of an error floor have been demonstrated earlier [32] using DRP interleavers. Similar results are demonstrated in this paper with QPP interleavers. 
The work in [1] opened the doors to a class of interleavers for turbo coding that provides the combination of an excellent performance, elegant algebraic and geometric analysis, and simplicity of implementation; to the best of our knowledge, there are no other interleavers sharing simultaneously these three characteristics to their fullest extent. Many problems in turbo coding that were earlier treated empirically may now be recast to be solved systematically and rigorously: the design of multiple turbo codes [33] for an improved asymptotic minimum distance by classifying the properties of PP interleavers under function composition; and puncturing of code bits for higher data rates using the $M C F$ theory [15] (a stronger generalization of an even-odd interleaver [13]) are only a couple of relevant examples.

\section{APPENDIX}

\section{NEW UPPER BOUND ON THE SPREAD FACTOR $D_{E}$}

A technique inspired from [22] is used to compute the upper bound $u b_{D_{E}}(N)$. We use the same distance $\delta$ in Section $\amalg$ but over the space $[0, N-1)^{2} \subset \mathbb{R}^{2}$ and forming the metric space $\mathcal{V}=\left([0, N-1)^{2}, \delta\right)$. In this metric space, a sphere of radius $r_{1}$ has the shape of a square when projected over the Euclidean metric space as shown in Fig. 8 If two spheres of radii $r_{1}$ and $r_{2}$ touch each other then the distance between their centers is $r_{1}+r_{2}$.

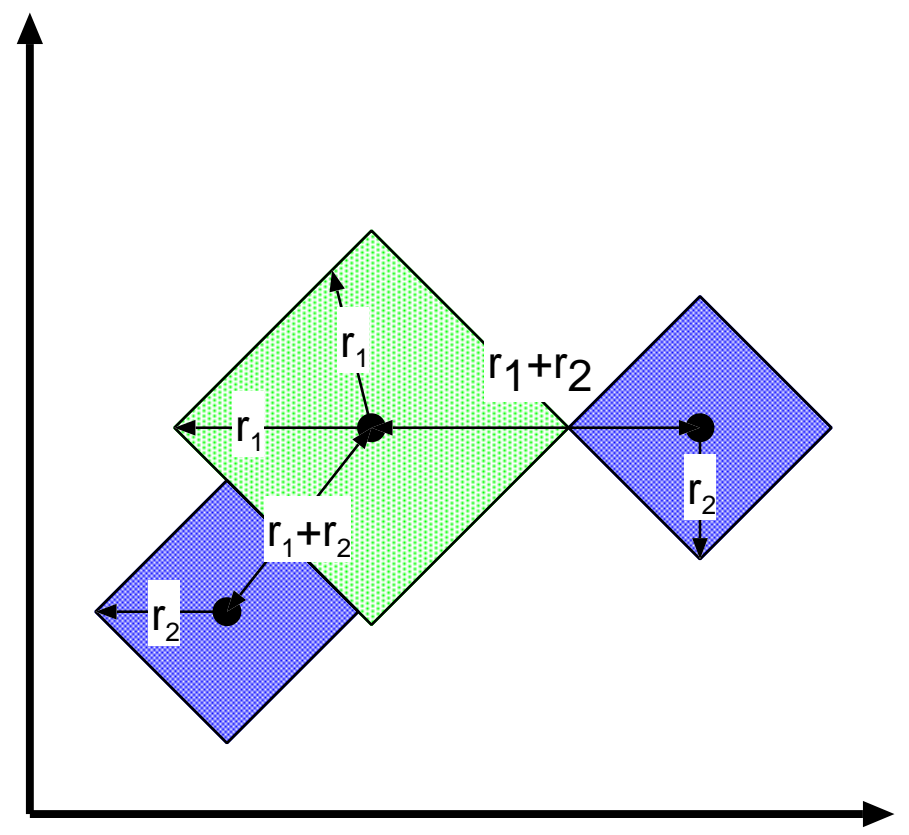

Fig. 8. Spheres in the metric space $(\mathcal{V}, \delta)$.

Before proceeding with the upper bound, it is straightforward to realize that in the metric space $\left(\mathbb{R}^{2}, \delta\right)$ there are only two types of densest packings for spheres with identical radius: aligned packing and unaligned packing as shown in Fig. 9 The unaligned packing is obtained by arbitrarily "sliding" stripes of the aligned packing.

The upper bound on $D_{E}$ is constructive. Given a square whose area is $(N-1)^{2}$, we attempt to pack $N$ spheres of identical radii $r$ and maximize the radii of the spheres $r$. We do so for values of $N$ for which clearly a subset of the aligned packing gives the densest packing as shown in Fig. 10. We end up with 1) several points touching the boundaries of $\mathcal{V}$ and 2) excess cover areas.

There are two classes of values for $N$. The cases $N=8$ and $N=18$ belong to

$$
N=2 p^{2}, \quad p=2,3,4 \ldots
$$




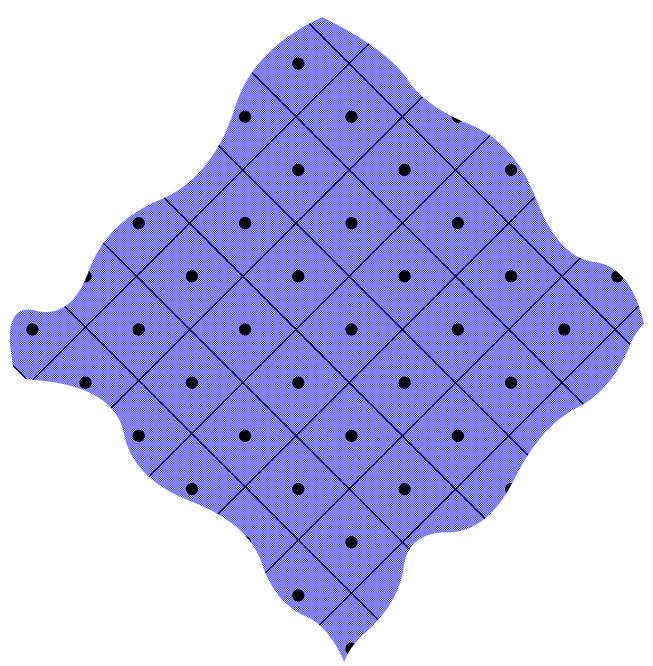

Aligned Packing

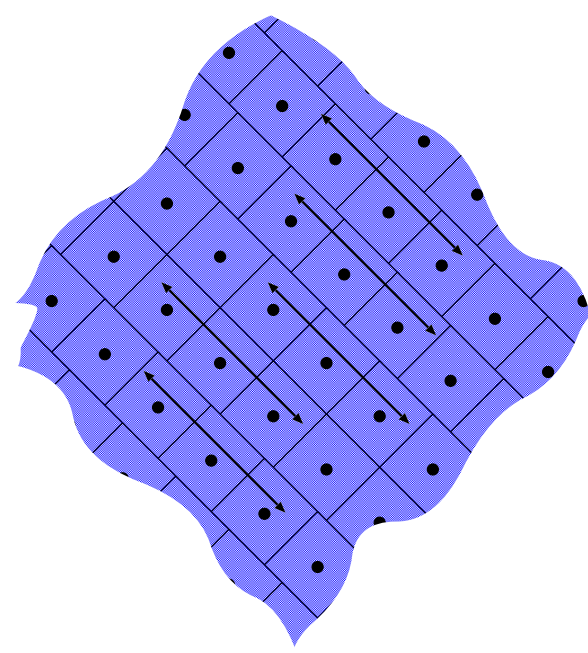

Unaligned Packing

Fig. 9. Sphere packing over the metric space $\left(\mathbb{R}^{2}, \delta\right)$.
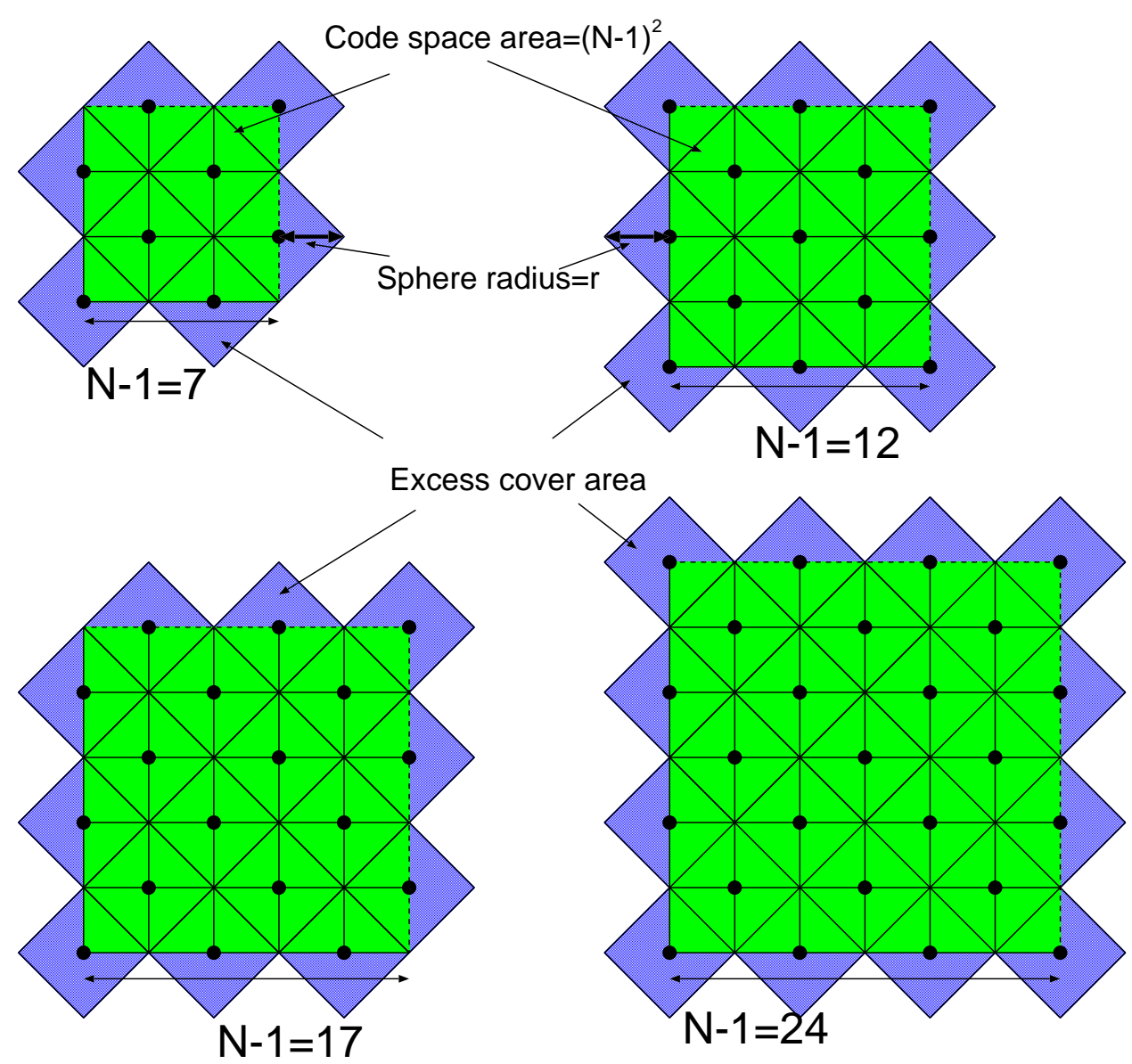

Fig. 10. Sphere packing over the metric space $(\mathcal{V}, \delta)$. 
where $p$ is the number of points on the bottom edge. The upper bound is the distance between adjacent points, i.e., $u b_{D_{E}}(N)=(N-1) /(2 p-1)$. After some simple algebra, the upper bound $u b_{D_{E}}(N)$ is

$$
u b_{D_{E}}(N)=\frac{2(N-1)}{\sqrt{2 N}-1}
$$

The cases $N=13$ and $N=25$ belong to

$$
N=p^{2}+(p-1)^{2}, \quad p=2,3,4, \ldots
$$

The upper bound $u b_{D_{E}}(N)$ is

$$
u b_{D_{E}}(N)=\frac{2(N-1)}{\sqrt{2 N-1}-1} .
$$

\section{A GeOMETRic APPROACH FOR MAXIMUM-SPREAD LINEAR INTERLEAVERS}

Although not explained in [12], it is clear that many candidates for an interleaver length $N$ achieving maximum-spread $\sqrt{2 N}$ are of the form $N=2 n^{2}$ because those allow the optimal packing in $\left(\mathcal{V}, \delta_{N}\right)$. This is illustrated in Fig. 11 for $N=32$. The dots show the potential places that may be occupied by a point of the interleaver-code. Without loss of generality, we place first a point in $(0,0)$. Next, we place a point along the line $-x+8(\bmod 32)$ and $0<x<8$ with the constraint that a linear curve that interpolates the first two points is a permutation polynomial. Then we follow a similar but simpler technique as we do to prove Theorem 2 in Appendix III. This leads to an alternate geometric construction for all maximum-spread linear interleavers (up to symmetries).

The authors of [8] explain the so-called dithered diagonal construction using the same geometric approach. The difference with linear interleavers is that the placement of a second point does not fix all the remaining points. Therefore a different slide (or dither) is allowed for the other stripes provided that an interleaver constraint is maintained.

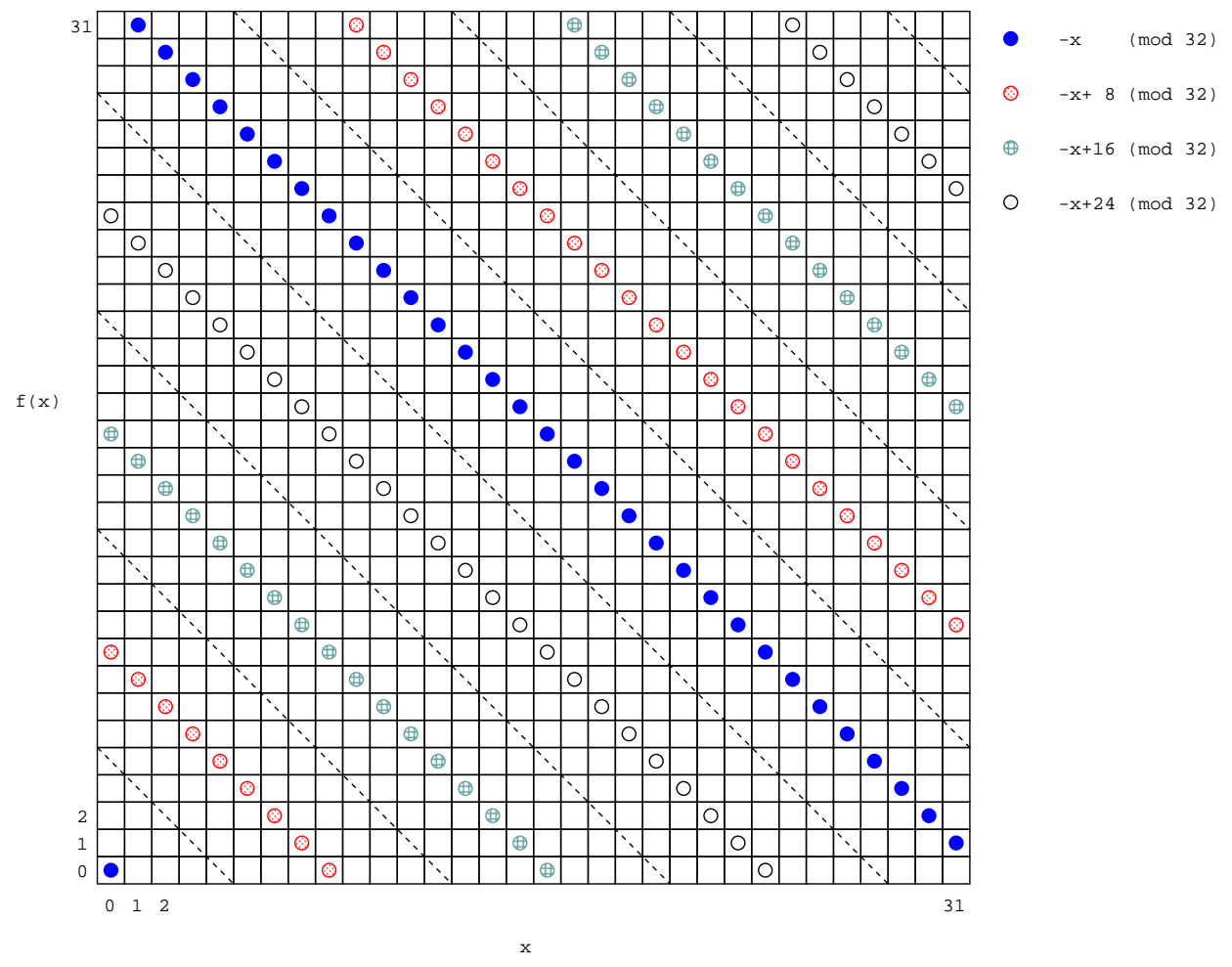

Fig. 11. Linear curves describing the "centers" of stripes in a perfect packing for $N=32$. 


\section{Proof of Theorem 2}

The set of linear curves $b_{i}(x)=-x+i 2 n(\bmod N), \quad 0 \leq i<n$ is simply passing through the center of the stripes in either the aligned or an unaligned packing as show in Fig. 11, i.e., the smallest distance $\delta_{N}$ between two points that belong to any two disjoint curves is $2 n$. If we show that the intersection of each of the curves $b_{i}(x)$ with $f(x)$ has $N / n$ solutions that are equally spaced by $\delta_{N}=2 n$ then we are done.

We start by rewriting the infinite sequence (4) as function of $n=2^{k-1}$.

$$
\left(2^{k}-1\right) x+2^{k+1} x^{2} \equiv(2 n-1) x+8 n x^{2} \quad\left(\bmod 2^{2 k-1}=2 n^{2}\right)
$$

The intersection of $(8)$ and a linear curve $b_{i}(x)$ is the solution to

$$
(2 n-1) x+8 n x^{2} \equiv-x+i 2 n \quad\left(\bmod 2 n^{2}\right),
$$

or

$$
2 n(\underbrace{-i+x+4 x^{2}}_{j}) \equiv 0 \quad\left(\bmod 2 n^{2}\right) .
$$

We then transform the quadratic congruence (9) into a linear congruence

$$
2 n j \equiv 0 \quad\left(\bmod 2 n^{2}\right),
$$

whose set of solutions is

$$
j \in\{0, n, 2 n, 3 n, \ldots,(2 n-1) n\},
$$

i.e., we have $2 n=N / n$ solutions that are equally spaced for (10). To solve (9), we simply need to find the solution to

$$
-i+x+4 x^{2} \equiv j \quad\left(\bmod 2 n^{2}\right),
$$

when $i$ and $j$ are fixed. Observing that $4 x^{2}+x\left(\bmod 2 n^{2}\right)$ is a QPP, the solution for $x$ is unique. Further, from the proof of the $M C F$ property of permutation polynomials [15, Theorem 1], we know that if $M$ divides $N$ then a set of $M$ equally spaced (modulo $N$ ) values by $N / M$ is always mapped to an equally spaced set of values by a PP and also by its inverse PP. Therefore we establish the desired result.

\section{Proof of Theorem 3}

If $\mathcal{A}\left(k_{0}, k_{1}\right)$ is an isometry of the interleaver-code $Q=\Phi(q)$ then we must have $q\left(x-k_{0}\right)+k_{1} \equiv q(x)$ $(\bmod N)$. Developing it we have

$$
\begin{gathered}
q_{2}\left(x-k_{0}\right)^{2}+q_{1}\left(x-k_{0}\right)+k_{1} \equiv q_{2} x^{2}+q_{1} x \quad(\bmod N) \\
q_{2} x^{2}+\left(q_{1}-2 q_{2} k_{0}\right) x-q_{1} k_{0}+q_{2} k_{0}^{2}+k_{1} \equiv q_{2} x^{2}+q_{1} x \quad(\bmod N)
\end{gathered}
$$

and therefore we just need to ensure $2 q_{2} k_{0} \equiv 0(\bmod N)$. This is a linear congruence and its solution is completely characterized in elementary number theory textbooks.

$$
k_{0}(i)=\frac{N i}{\operatorname{gcd}\left(2 q_{2}, N\right)}, \quad i=0,1,2, \ldots, \operatorname{gcd}\left(2 q_{2}, N\right)-1
$$


where $k_{0}(i)$ is the $i$-th solution and

$$
k_{1}(i)=q_{1} k_{0}(i)-q_{2} k_{0}(i)^{2}
$$

There are exactly $\operatorname{gcd}\left(2 q_{2}, N\right)$ distinct solutions, which means that each point in $Q$ belongs to an orbit of size $\operatorname{gcd}\left(2 q_{2}, N\right)$, i.e., the degree of shift-invariance is $\epsilon(Q)=\operatorname{gcd}\left(2 q_{2}, N\right)$. Hence, from Proposition 5 the degree of non-linearity for QPPs is $\zeta(Q)=N / \operatorname{gcd}\left(2 q_{2}, N\right)$.

\section{REFERENCES}

[1] J. Sun and O. Y. Takeshita, "Interleavers for turbo codes using permutation polynomials over integer rings," IEEE Trans. Inform. Theory, vol. 51, no. 1, pp. 101-119, Jan. 2005.

[2] A. Nimbalker, T. K. Blankenship, B. Classon, T. E. Fuja, and D. J. Costello, Jr., "Contention-free interleavers," in Proc. 2004 IEEE International Symposium on Information Theory, Chicago, IL, 2004, p. 54.

[3] C. Berrou, S. K. Y. Saouter, C. Douillard, and M. Jézéquel, "Designing good permutations for turbo codes: towards a single model," in Proc. International Conference on Communications, vol. 1, Paris, France, June 2004, pp. 341-345.

[4] C. J. Corrada-Bravo and I. Rubio, "Deterministic interleavers for turbo codes with random-like performance and simple implementation," in Proc. 3rd International Symposium on Turbo Codes, Brest, France, Sept. 2003.

[5] A. Giulietti, L. van der Perre, and M. Strum, "Parallel turbo coding interleavers: avoiding collisions in access to storage elements," IEE Electronics Letters, vol. 38, no. 5, pp. 232-234, Feb. 2002.

[6] H. R. Sadjadpour, N. J. A. Sloane, M. Salehi, and G. Nebe, "Interleaver design for turbo codes," IEEE J. Select. Areas Commun., vol. 19, no. 5, pp. 831-837, May 2001.

[7] O. Y. Takeshita and D. J. Costello, Jr., "New deterministic interleaver designs for turbo codes," IEEE Trans. Inform. Theory, vol. 46, no. 6, pp. 1988-2006, Sept. 2000.

[8] S. Crozier, "New high-spread high-distance interleavers for turbo-codes," in Proc. 20th Biennial Symposium on Communications, Kingston, Ontario, Canada, May 2000, pp. 3-7.

[9] S. Crozier and P. Guinand, "High-performance low-memory interleaver banks for turbo-codes," in Proc. 54th IEEE Vehicular Technology Conference (VTC 2001 Fall), Atlantic City, New Jersey, Oct. 2001, pp. 2394-2398.

[10] F. Daneshgaran and M. Mondin, "Design of interleavers for turbo codes: iterative interleaver growth algorithms of polynomial complexity," IEEE Trans. Inform. Theory, vol. 45, no. 6, pp. 1845-1859, Sept. 1999.

[11] O. Y. Takeshita and D. J. Costello, Jr., "On deterministic linear interleavers for turbo-codes," in Proc. 35th Annual Allerton Conference on Communication, Control, and Computing, Sept. 1997, pp. 711-712.

[12] S. Dolinar and D. Divsalar, "Weight distribution of turbo codes using random and nonrandom permutations," JPL, TDA Progress Report 42-122, Aug. 1995.

[13] A. S. Barbulescu and S. S. Pietrobon, "Interleaver design for turbo codes," IEE Electronics Letters, vol. 30, no. 25, pp. 2107-2108, Dec. 1994.

[14] C. Berrou, A. Glavieux, and P. Thitimajshima, "Near Shannon limit error-correcting coding and decoding: turbo-codes," in Proc. IEEE Int. Conf. on Commun., Geneve, Switzerland, May 1993, pp. 1064-1070.

[15] O. Y. Takeshita, "On maximum contention-free interleavers and permutation polynomials over integer rings," IEEE Trans. Inform. Theory, Mar. 2006, to be published. [Online]. Available: http://arxiv.org/abs/cs.IT/0506093/

[16] J. Ryu and O. Y. Takeshita, "On quadratic inverses for quadratic permutation polynomials over integer rings," IEEE Trans. Inform. Theory, Mar. 2006, to be published. [Online]. Available: http://arxiv.org/abs/cs.IT/0511060/

[17] M. Cheng, M. Nakashima, J. Hamkins, B. Moision, and M. Barsoum, "A decoder architecture for high-speed free-space laser communications," in Proc. of SPIE, vol. 5712, Apr. 2005, pp. 174-185.

[18] O. Y. Takeshita, "A new construction for LDPC codes using permutation polynomials over integer rings," IEEE Trans. Inform. Theory, submitted for publication. [Online]. Available: http://arxiv.org/abs/cs.IT/0506091/

[19] R. L. Rivest, "Permutation polynomials modulo $2^{w}$," Finite Fields and their Applications, vol. 7, pp. 287-292, Feb. 2001.

[20] C. Y. Lee, "Some properties of nonbinary error-correcting codes," IRE Trans. Inform. Theory, vol. 4, no. 2, pp. 77-82, June 1958.

[21] M. Breiling, "A logarithmic upper bound on the minimum distance of turbo codes," IEEE Trans. Inform. Theory, vol. 50, no. 8, pp. 1692-1710, Aug. 2004.

[22] E. Boutillon and D. Gnaedig, "Maximum spread of D-dimensional multiple turbo codes," IEEE Trans. Commun., vol. 53, no. 8, pp. 1237-1242, Aug. 2005.

[23] B. Moision and M. Klimesh, "Some observations on permutation polynomials," JPL Inter-office Memorandum 331.2005.1.1.

[24] G. D. Forney, Jr., "Geometrically uniform codes," IEEE Trans. Inform. Theory, vol. 37, no. 5, pp. 1241-1260, Sept. 2001.

[25] P. M. Neumann, G. A. Stoy, and E. C. Thompson, Groups and Geometry. Oxford University Press, 1994.

[26] E. Rosnes and $\varnothing$. Ytrehus, "Improved algorithms for the determination of turbo-code weight distributions," IEEE Trans. Commun., vol. 53, no. 1, pp. 20-26, Jan. 2005.

[27] C. Weiss, C. Bettstetter, and S. Riedel, "Code construction and decoding of parallel concatenated tail-biting codes," IEEE Trans. Inform. Theory, vol. 47, no. 1, pp. 366-386, Jan. 2001.

[28] P. Guinand and J. Lodge, "Trellis termination for turbo encoders," in Proc. 17th Biennial Symposium on Communications, Kingston, Ontario, Canada, May 1994, pp. 389-392.

[29] J. Gathen and J. Gerhard, Modern Computer Algebra. Cambridge University Press, 1999.

[30] R. Garello, P. Pierloni, and S. Benedetto, "Computing the free distance of turbo codes and serially concatenated codes with interleavers: algorithms and applications," IEEE J. Select. Areas Commun., vol. 19, no. 5, pp. 800-812, May 2001. 
[31] “The 3rd generation partnership project (3GPP)," 2005. [Online]. Available: http://www.3gpp.org/

[32] S. Crozier and P. Guinand, "Distance upper bounds and true minimum distance results for turbo-codes designed with DRP interleavers," in Proc. 3rd International Symposium on Turbo Codes \& Related Topics, Brest, France, Sept. 2003, pp. 169-172.

[33] D. Divsalar and F. Pollara, "Multiple turbo codes for deep-space communications," JPL, TDA Progress Report 42-121, May 1995. 